\title{
A spectral density approach for modelling continuous vertical forces on pedestrian structures due to walking
}

JMW Brownjohn

Associate Professor, School of Civil and Environmental Engineering, Nanyang Technological University, Singapore

A Pavic

Senior Lecturer in Structural Engineering, Department of Civil and Structural Engineering, University of Sheffield, UK

P Omenzetter

Research Fellow, School of Civil and Environmental Engineering, Nanyang Technological University, Singapore

Contact author: $\quad$ Dr James Brownjohn

School of Civil and Environmental Engineering N1-1a-11

50 Nanyang Avenue

Singapore 639798

Singapore

e-mail: cjames@ntu.edu.sg 


\begin{abstract}
A limitation of existing walking models used for vibration serviceability assessment of structures carrying pedestrians is that they are typically based on direct measurements of single footfalls replicated at precise intervals. This assumption of 'perfect periodicity' allows walking forces to be modelled as a Fourier series of sine waves having frequencies of the walking pace and its integer multiples. The true imperfection and randomness in walking is currently not taken into account even in more advanced dynamic loading codes of practice and leads to an unknown degree of conservatism.
\end{abstract}

Having this in mind, this paper examines real continuous walking forces obtained from an instrumented treadmill and the effect of their random imperfection through time and frequency domain simulations of structural response.

The main conclusions are that there are significant differences between responses due to the imperfect real walking forces and the 'equivalent' perfectly periodic simulation. These differences are most significant for higher harmonics where the simulated vibration response overestimates the real-life behaviour, sometimes significantly. This is mainly due to random imperfections in real walking. Given that a more realistic representation of imperfect walking is an auto-spectral density function, the random character naturally leads to a stochastic approach to treatment of pedestrian loading applied in the frequency domain. The approach can be used for single pedestrians but the 
benefits are greater for crowd loading where correlation between pedestrians as well as statistics of their pacing rates can be applied directly.

Key words: coherence, crowd loading, footbridges, gait analysis, long-span floors, pedestrian loading, spectral density, walking forces 


\section{Introduction}

An increasing interest in vibration serviceability of slender civil engineering structures has led to a requirement for better understanding of footfall forces generated by pedestrians. This interest of structural engineers has been preceded by many year of biomechanics research into ground reaction forces on the human musculo-skeletal system (Winter 1990). Structural engineers have begun to use data from these studies as well as from their own direct or indirect measurements with an aim to develop realistic models of human footfall forces. These are then employed in vibration serviceability assessments of structures occupied and dynamically excited by pedestrians, such as footbridges and long span floors.

Numerous models of walking excitation have been proposed (Pavic and Reynolds 2001; Bachmann and Ammann 1987) and usually derived from the assumption that walking is a perfectly periodic activity. The simplest such model that uses a single sinusoidal function is found in current British (BSI 2001) and Canadian (CSA 2000) codes. This means that actual forces due to continuous walking can be re-created, by adding a sequence of identical single footfall traces, temporally displaced by integer multiples of an exact footfall interval. The footfall forces are obtained by walking over a 'neutral' force plate that is supposed not to affect the walking pattern. The idea is illustrated in Figure 1 where identical left and right foot time histories are shown separately and added, resulting in a synthetic walking force time history. In a time domain analysis procedure that uses a synthetic walking time series, the structure is assumed to be linearly elastic and, using modal decomposition, the response of each vibration mode can be analysed separately 
using harmonic loads modulated by the appropriate mode shape to account for the moving load (Smith 1988).

Using Fourier analysis, any perfectly periodic and continuous signal $x(t)$ repeating at intervals $T$ can be represented by an infinite $(m \rightarrow \infty)$ sum of terms:

$$
x(t)=a_{0}+\sum_{n=1}^{m} a_{n} \cdot \sin \left(2 \pi n / T+\phi_{n}\right)
$$

Hence if we take a sample of duration $T$ [s] from a synthetic time series for walking at a pacing rate of $\bar{f}=1 / T$ footfalls per second $[\mathrm{Hz}]$ the walking forcing function can be decomposed, via equation (1), into:

- a mean value $a_{0}$, equivalent to body weight, in the case when $x(t)$ presents a force induced by a pedestrian,

- a fundamental component $(n=1)$ at pacing rate $\bar{f}$, and

- a sequence of $m-1$ higher harmonics, where the value of $m$ depends on which model is used, and inclusion of the sixth harmonic is not unusual (Ellis 2000). 
It should be noted that $\phi_{n}$ are phase angles which have been found to vary considerably between various measurements for higher harmonics (Bachmann et al. 1995) indicating the randomness in the process of generation of dynamic forces due to human walking. However, little utilisation of this important finding was made to date. The values of normalised coefficients $G_{n}=a_{n} / a_{0}$ are known as 'dynamic load factors' (DLFs) and depend on walking speed and individual characteristics of a human test subject. DLFs for walking, running and jumping are presented by Rainer et al. (1988) for three subjects, while a more comprehensive study of walking has been undertaken by Kerr (1998). His study found wide variability of DLFs between subjects participating in the tests (intersubject variability) and also between different tests with the same subject (intra-subject variability). Hence it is appropriate to give a frequency-dependent mean and coefficient of variation for DLFs, as recently proposed by Young (2001) based on Kerr's data.

The study presented in this paper describes results of measurements performed by the authors using an instrumented treadmill. The walking forcing function was therefore measured in a continuous manner which provided an opportunity to study their randomness. The effects of the stochastic nature of human walking on the calculated structural response were considered by comparing the stochastic responses with their counterparts obtained by using a perfectly periodic excitation model which is nowadays frequently utilised in practice. The perfectly periodic model of Fourier coefficients lends itself to deterministic time domain analysis, while the dispersed energy seen for real continuous walking is more suited to the techniques of stochastic analysis in frequency 
domain, where the walking forces are presented as auto-spectral densities (ASDs), (McConnell 1995).

When applied with the Gaussian form of probability distribution of mean pacing rates, application of ASDs leads to a framework for more realistic frequency domain representation for large numbers of pedestrians. Additionally, the consideration of correlation between pedestrians at different locations on a structure suggests an approach similar to estimating dynamic response of structures to turbulent buffeting by wind. Finally, an example of bridge response to large numbers of pedestrians is presented as evidence to support the proposed analytical framework.

\section{Frequency domain modelling of walking forces}

The significance of imperfect periodicity in dynamic loads induced by pedestrians on footbridges has been shown, for example, by Rainer et al. (1988). The sinusoidal steadystate SDOF response models they used for footbridge vibration serviceability assessment were based on walking harmonics exciting the first mode resonance. These analytical models predicted peak accelerations which were almost twice as high as experimental values produced by even the best attempts to excite resonance with perfectly timed walking.

A relatively small number of studies (Eriksson 1994; Mouring and Ellingwood 1994) have considered predicting response using frequency domain models. For example, Mouring and Ellingwood (1994) generated 'random' time domain histories of crowd 
loading $p(t)$ from simulations of different arrival times of individual walkers and their pace rates. These were then converted into generalised modal forces $p^{\prime}(t)$ via a known mode shape and further transformed, via the single-sided discrete Fourier transform (DFT), into Fourier (amplitude) spectra $P^{\prime}(f)$ having spectral line spacing $d f$, where $f$ $[\mathrm{Hz}]$ is frequency. The ASD $S_{P}^{\prime}(f)$ of modal loading could be obtained from such single sided Fourier spectra via the following formula:

$$
S_{P}^{\prime}(f)=P^{\prime 2}(f) /(2 d f)
$$

Note that when plotted on linear axes, ASDs are often shown in square root form $\sqrt{S_{P}^{\prime}(f)}$, which is defined here as 'root ASD'.

Given the system frequency response function $H(f)$ for the mode, the variance of modal response $X(f)$ in a given frequency band $f_{1}-f_{2}$ around a specific vibration mode could be obtained via (Newland 1993):

$$
\sigma_{X, f_{1}-f_{2}}^{2}=\int_{f_{1}}^{f_{2}} S_{P}^{\prime}(f)|H(f)|^{2} d f
$$


As the individual forcing time histories in Mouring and Ellingwood's study were assumed to be perfectly periodic sums of sinusoids, the approach considered only one aspect of randomness of walking. The fundamental issue of imperfect individual forcing functions was not accounted for.

\section{Measurement and analysis of real continuous walking forces}

Forces generated during walking on a structure are ideally determined by direct measurement of the contact force while walking on the structure itself, as account must be taken of the surface conditions and of the response of the structure, which may modify the footfall forces (Dallard et al. 2001). While force plate measurements on a structure during jumping are a practical means to measure directly the applied loading as a sequence of footfalls, measurement of continuous walking forces requires a sensor that moves with the walker, a fully instrumented walkway, or a treadmill.

Instrumentation for measuring contact forces during continuous walking

Matsumoto et al. (1978) appear to have made an early attempt to recover continuous forces through measurements of acceleration at the waist of pedestrians walking across a stiff floor. However, an erroneous assumption that human frame behaves as a rigid body was required. Some improvement on this approach could be obtained using movies or video recordings of human motion (Winter 1990; Forner Cordero et al. 2002). Devices for direct measurement of forces during walking have been developed, for example, in the form of in-sole pressure sensors (Woodburn and Helliwell 1996; Barnett et al. 2001; Razian and Pepper 1998). 
A rare study using an instrumented laboratory-based walkway (Ebrahimpour et al. 1996) has provided valuable information about continuous pacing footfall forces and probably the most relevant guidance on the effect of multiple pedestrians, but alas there was an inevitable limit to the length of the walkway.

Treadmills capable of measuring three orthogonal components of footfall force are now available (Belli et al. 2001), but those measuring only the vertical component are more common. The Biomechanics Laboratory at the National Institute of Education (NIE) in Singapore has a 'Gaitway' (Rao et al. 2002), shown in Figure 2. This is a conventional exercise treadmill equipped with a pair of force plates under the belt fore and aft of the mid position that should also be the optimal location of the test subject. A reflective marker attached to the near foot of a test subject (Figure 2) is used as a trigger to help software retrieve left and right foot force time series from the fore and aft force plate signals.

$\underline{\text { Test sequence and continuous walking statistics for three test subjects }}$

A series of measurements were made using the NIE Gaitway to characterise walking forces for three test subjects walking at a range of speeds: Subject P is a $65 \mathrm{~kg}$ male researcher, Subject K is a $62 \mathrm{~kg}$ male student and Subject Y is a $46 \mathrm{~kg}$ female student. All were asked to walk with a comfortable range of walking speeds from $2.5 \mathrm{~km} / \mathrm{h}$ to $7.5 \mathrm{~km} / \mathrm{h}$ (note: $3.6 \mathrm{~km} / \mathrm{h}=1 \mathrm{~m} / \mathrm{s})$. 
Each test comprised a sequence of measurements of approximately one minute of steady walking with a fixed treadmill speed. It should be stressed here that the pacing was not prompted by any device such as a metronome, and pacing rate was determined only from subsequent analysis of the signals.

It could be argued that when walking as a crowd or small group, pedestrians will often be forced to maintain their speed at a certain level to stay with the flow. Hence, these data may help provide quite realistic representations of walking under such circumstances of controlled walking speed.

The relationship between pacing rate and walking speed is known to vary between subjects. For example, Subject K's long stride allowed him to reach speeds that Subject P could not achieve without running. This inter-subject variation contributes to the Gaussian distribution of walking pedestrian rates reported, for example, by Matsumoto et al. (1978), but the intra-subject variations for a single pedestrian are of greater interest here.

The Gaitway system is normally used to provide a series of statistics or 'gait parameters', describing footfalls. Gait analysis is a well established research area (Rao et al. 2002) for studying dynamics of the human body and, more recently, via pattern recognition, as a tool for identifying individuals by the way they walk (Stevenage et al. 1998). Even so, data relevant for structural analysis concerning intra-subject variations in timing of the 
individual footfalls (i.e. the variations of the footfall interval, as annotated in Figure 1) and their impulses (top of Figure 1), are not readily available from the literature.

For Subjects K, P and Y, Figure 3 shows mean values of footfall (i.e. pulse) width and of normalised impulse, both as functions of the mean pace rate. The normalised impulse is defined as the footfall impulse divided by test subject body weight. The obvious trend for footfall width and normalized impulse to reduce with increasing pacing rate is logical, but the low scatter between subjects was not expected. Nevertheless, the coefficients of variation (COVs) of these two gait parameters established by analysing one-minute blocks of measured data were used to quantify the imperfection of walking. $\operatorname{COV} \rho$ is defined as:

$$
\rho=\frac{\sigma}{\mu}
$$

where $\sigma$ is the standard deviation and $\mu$ is average value of a gait parameter, each obtained using the same one-minute walking sample with mean pacing frequency $\bar{f}$. Analysis of data from all three test subjects established that at each mean pacing frequency, COVs for pulse width and normalized impulse are typically close to $2 \%$, while for the interval between footfalls the COV is closer to $3 \%$. There seems to be no trend with these COV values, which also show no minimum value that would indicate an optimal comfortable walking pattern. These small variations show that walking has an inherent degree of randomness, whose effect could be studied using a frequency domain 
approach, as used in random vibration analysis. The effect of these small variations is illustrated through numerical examples in the next section, after which an analytical framework is proposed for the development of more realistic and less conservative procedures to estimate structural vibration response to walking.

\section{Quantification of structural response to real walking}

For structures experiencing continuous walking forces due to a crowd of individual pedestrians who arrive on and eventually leave the structure according to a Poisson distribution (Matsumoto et al. 1978), each individual contributes a transient build up and decay of the structural dynamic response. In this process it is assumed that a kind of random but stationary, state is achieved. Alternatively, we can examine the quasi steadystate structural response to a single imperfect pedestrian after the initial transient build up. In either circumstance, where stationarity (Newland 1993) of the structural response can be achieved, equation (3) can be applied. Firstly, the case of the single pedestrian is examined.

Figure 4a shows the measured force time series for Subject $\mathrm{P}$ walking at $4.4 \mathrm{~km} / \mathrm{h}$ at a mean pace rate of $\bar{f}=1.91 \mathrm{~Hz}$ and Figures $4 \mathrm{c}-\mathrm{f}$ show response to this force for $1000 \mathrm{~kg}$ oscillators with $1 \%$ damping and natural frequencies $f$ of $1.91 \mathrm{~Hz}, 3.82 \mathrm{~Hz}, 5.73 \mathrm{~Hz}$ and $7.64 \mathrm{~Hz}$. These frequencies correspond exactly to the fundamental and higher harmonics of the walking excitation. 
The real force time history of Figure 4 represents a segment of 30 paces extracted from one $60 \mathrm{~s}$ walking force time history. An average footfall was obtained from the full trace, by averaging amplitudes of all footfalls using a time base with a trigger point at the beginning of the initial steep rise of each footfall. A synthetic and perfectly periodic time history was then generated by replicating this average footfall, in the manner presented in Figure 1. Figure $4 \mathrm{~b}$ shows the resulting 30-pace time history. The envelopes of maxima and minima of response of the same set of four $1000 \mathrm{~kg}$ oscillators to this time series are superimposed on the response to real walking shown in Figures 4c-f for comparison.

There is little to choose between the two responses for the oscillator when its natural frequency exactly equals the mean pacing rate $(f=\bar{f})$, which explains why it is often possible to build up to a significant percentage of resonant response to the fundamental component of the walking force even with imperfect walking. Although the duration is not long enough to detect any difference in the 'steady state' response, the maximum response is only a little less for the real walking.

However, it should be noted that when the pacing of a pedestrian becomes out of phase with the vibration of a footbridge structure excited close to resonance, energy is extracted from the structure and the human exciter becomes an active damper. Pimentel et al. (2001) noted this phenomenon with pedestrians exciting very lively footbridges in antisymmetric vertical modes. 
For the second, third and fourth harmonics, the difference is much clearer, particularly for the second harmonic which is somewhat surprising. After the transient build up to a significant proportion of resonant maximum amplitude, the response fades. For these harmonics, on average, the SDOF systems have greater response under the excitation of a perfect 'averaged' walking. However, it is worthwhile noticing that occasionally the response due to real imperfect excitation exceeds its synthetic counterpart.

In the literature on vibration serviceability there are two common measures of the structural response signals: the signal absolute peak value and some form of the signal 'average' amplitude. The former is self explanatory, the latter involves determining the variance of the signal, which is the mean square of a signal $a(t)$ with the mean value (also known as the DC component) removed. This variance $\sigma_{a}^{2}$ can be computed from either $a(t)$ or its ASD $S_{A}^{\prime}(f)$. The root mean square $(R M S)$ of a signal is another common measure based on the mean square of the signal but including the DC component. Where the DC component is zero, as for example in acceleration signals, we have $\operatorname{RMS}(a)=\sigma_{a}$.

The relationship between peak value $\hat{a}$ and the variance $\sigma_{a}^{2}$ is given by the 'crest factor' (CF) defined as (McConnell 1995):

$$
C F=\frac{\hat{a}}{\sigma_{a}}
$$


The crest factor for a steady state sine is exactly $\sqrt{2}$ while for random signals it typically ranges from 3 to 5. In wind engineering (Simiu and Scanlan 1996) a crest factor of 3.7 is used typically to obtain peak gust speeds from mean $R M S$ wind speeds.

The shapes of acceleration responses in Figure 4 show that the RMS of real walking responses is usually lower compared with the synthetic counterparts. However, the crest factors are apparently higher for real walking, with the clear possibility that the corresponding peak values may be larger compared with responses to synthetic walking. This is a standard feature of random vibrations and emphasises the stochastic character of real walking.

The different levels of response due to real and synthetic walking can be studied in a more systematic way by computing the $R M S, \sigma_{a}$ and maximum peak of response for SDOF oscillators corresponding to each harmonic during the 'steady state' part of the response after the initial build up. Longer data sets of 90 paces are used to obtain more reliable RMS values and show the significance of crest factor. It should be noted that 90 paces of the slowest walking lasts just over one minute.

To allow for the possibility that the $R M S$ and maximum peak response values for each harmonic do not occur at frequencies which are exact multiples of $\bar{f}$, a response spectrum approach is used. Figure 5 (upper) shows plots of loci of peak values of acceleration for low-damped $(\zeta=1 \%)$ oscillators having natural frequencies in the 
narrow range of frequencies centred on $\bar{f}$. Figure 5 (lower) shows the corresponding $R M S$ values. The thick and thin lines, respectively, indicate the spectra for real and synthetic walking, and the differences in Figure 4 are more clearly apparent in Figure 5.

Figures 4 and 5 apply to a single walking sample. Similar simulations were performed using measured forcing functions from other tests and the results are best summarised by taking, for each time series, the maximum value around each walking harmonic (see Figure 5), and plotting them against $\bar{f}$ for measured and synthetic continuous walking. Figure 6 shows these maximum $R M S$ values for each harmonic and Figure 7 shows the maximum peak values. With very few exceptions, for every harmonic at every pacing rate, $R M S$ response is less for the real walking, with the difference being most significant for the second harmonic. This confirms the previous observation made when visually examining and comparing the sample represented by Figure $4 \mathrm{~d}$.

For the fundamental harmonic component, the peak response values corresponding to real forcing function are similar to their synthetic counterparts, but for higher harmonics they are generally higher for real walking. Lower $R M S$ values but higher peak values correspond to higher crest factors, whose average values for each harmonic are given in Figure 6 as $\mathrm{CF}(\mathrm{r})$ for the real and $\mathrm{CF}(\mathrm{s})$ for the synthetic walking force inputs. For the fundamental harmonic, $\mathrm{CF}(\mathrm{r})$ is close to the crest factor corresponding to steady state sine value while for real walking in higher harmonics the crest factors approach values used in random data analysis. The same trend was observed to differing degrees for all three test 
subjects. However, further parametric studies demonstrated that the differences reduce with increase in damping of the SDOF systems studied.

This inaccuracy of the conventional time domain approach using DLFs has been commented on in the published literature (Pavic and Reynolds 2002), and the remainder of the paper is focused on providing and validating a more realistic and less conservative approach.

\section{Frequency domain modelling of imperfect real walking}

In the context of vibration serviceability, it is nowadays usual to use $R M S$ values of structural vibration response (typically accelerations) rather than peak values as criteria for assessment (Gordon 1991; BSI 1992). With the exercise just described in mind, this has two consequences. Firstly, it can be seen that computation of response based on assumed perfect walking will be conservative, especially for higher harmonics. Secondly, by virtue of equation (3) it is clear that the effective reduction of the $R M S$ response could be modelled by a more realistic shaping of the ASD of the walking forcing function $S_{P}^{\prime}(f)$.

For example, a SDOF oscillator with $1 \%$ damping excited at resonant frequency by a sinusoidal forcing function with amplitude $\sqrt{2}[\mathrm{~N}]$ will have $R M S$ response 50 times the static displacement $\bar{x}$. Where the input signal is not a perfect sine but 'leaks' energy into adjacent frequencies on either side of the resonance, the $R M S$ of response obtained in the manner of equation (3) will then be less than $50 \bar{x}$. This 'leaking' of the excitation energy 
is a key reason for overestimation of $R M S$ levels due to real walking when synthetic walking forcing functions are used (Figure 6).

\section{Fourier spectra of continuous walking}

Figure 8 shows the discrete Fourier line spectra of exactly 64 paces of a measured continuous walking force time series. To avoid leakage during the digital signal analysis, the duration of the whole data block was fixed at 64 times the mean interval $T$ between footfalls where $T=1 / \bar{f}$. The amplitudes of the lines do not represent spectral densities, but simply amplitudes of the elements of the Fourier series at successive discrete spectral lines spaced at intervals of $\bar{f} / 64$. Likewise Figure 9 shows the same analysis applied to the synthetic continuous walking.

In both graphs the amplitude values have been normalised with respect to body weight $W$, so in Figure 9 the amplitude of lines are in fact DLFs, as previously defined. Figure 9 illustrates how the perfect walking forcing function can be approximated with its first $m$ ( $m=10$ in Figure 9) components as:

$$
f(t) / W=1+\sum_{n=1}^{m} G_{n} \cdot \sin \left(2 \pi n \bar{f}+\phi_{n}\right)
$$

The above series can also be used to represent a range of human-induced excitation, such as running or jumping, and the values of $G_{n}$ are given in different forms in different 
research papers, codes of practice and design guides. For example, Young (2001)

proposed to calculate the DLF corresponding to the fundamental harmonic of walking as:

$$
G_{1}=0.37(\bar{f}-0.95)
$$

having in mind that, due to observed variability of the test data, there was a $17 \%$ coefficient of variation associated with this DLF. This COV is greater than observed in the tests on Subjects K, P and Y and it should be mentioned that Young's data were based on a much wider pool of tests and test subjects involving more than 800 single-footfall force measurements.

\section{'Leakage' of DLFs for real walking}

Comparison of Figures 8 and 9 shows a 'leaking' of energy from the perfectly periodic signal (Figure 9) to adjacent spectral lines in real walking (Figure 8), the effect being more pronounced for higher harmonics. The effect appears to vary more or less randomly between samples of the measured walking forcing function, reflecting the observations that COVs of stride width, interval and impulse are relatively insensitive to pace rate.

To observe the leakage effect across all pacing rates, the lines in the real walking spectra have first been normalised with respect to values of $G_{n}(n=1,2, \ldots, 6)$ obtained from corresponding synthetic spectra such as the one in Figure 9. This leads to the real walking spectra of the kind shown in Figure 8 corresponding to the frequency range where most 
of the energy is concentrated in each harmonic (assumed to be in the frequency range from $0.95 n \bar{f}$ to $1.05 n \bar{f}$ ). As the frequency spacing is proportional to $\bar{f}$, Fourier spectra for different pacing rates $\bar{f}$ have peaks at integer values $n$ on a normalised frequency axis $f / n \bar{f}$. Next, an $R M S$ average over all pacing rates and all test subjects may be taken by averaging over the squared ordinates around each harmonic. Figure 10 shows the resulting values of DLF $G_{n}^{\prime}(f / \bar{f})$ for which a good fit is obtained using the function:

$$
G_{n}^{\prime}(f / \bar{f})=A+B \exp \left(\frac{-|f / \bar{f}-1|^{C}}{D}\right)
$$

The curve-fitting parameters A, B C and D depend on the harmonic being approximated and are given in Table 1. As each ordinate in Figure 10 represents a Fourier amplitude, equation (2) can be used to obtain the corresponding ASD (given that $d \bar{f}=\bar{f} / 64$ ), as follows:

$$
S_{G_{n}^{\prime}}(f / \bar{f})=G_{n}^{\prime 2}(f / \bar{f}) / 2 d \bar{f}
$$

The leakage depends on the harmonic centre frequency - the higher the centre frequency - the greater the 'spread' of excitation energy. This is illustrated in Figure 11 where the frequency axis is expanded using $f / \bar{f}$ instead of $f / n \bar{f}$. 


\section{Models for frequency domain estimation of structural response to walking}

Procedures, or models, can now be established for the following two principal cases: a single 'imperfect' pedestrian, and a group of 'imperfect' pedestrians with a varying degree of correlation (from none to perfect) or synchronisation. For both cases there is currently considerable uncertainty and minimal guidance in design codes.

\section{$\underline{\text { Single 'imperfect' pedestrians }}$}

For a single person walking at a mean pace rate corresponding to the natural frequency of a floor or footbridge, in the first few cycles of vibration the imperfect pacing results in negligible reduction in the achieved response with respect to the perfect synthetic walking. However, as already shown, there is a difference when the walking lasts long enough to be treated as a (very) narrow band random process with root ASD following the shape of Figure 11 for each harmonic. Hence for an individual pedestrian the approach of equation (3) to determine the ASD and hence narrow band $R M S$ of response can be applied. The peak response values, if needed, can be determined from the calculated $R M S$ values via established crest factors (Figure 7).

\section{Groups of unsynchronised 'imperfect' pedestrians}

The distribution of $\bar{f}$ for a group of pedestrians could be assumed to be normal

(Bachmann and Amman 1987) and modelled by a probability density function $\phi(\bar{f})$

with a mean $\mu_{\bar{f}}$ and variance $\sigma^{2}{ }_{\bar{f}}$ : 


$$
\phi(\bar{f})=\frac{1}{\sqrt{2 \pi} \sigma_{\bar{f}}} \exp \left(-\frac{\left(\bar{f}-\mu_{\bar{f}}\right)^{2}}{2 \sigma_{\bar{f}}^{2}}\right)
$$

If individuals are assumed to walk with perfect periodicity, equation (10) gives the scaled shape of the ASD of walking forces for a crowd.

For each individual $i$ with mean pacing rate $\bar{f}_{i}$ the ASD of loading is itself described via a (spectral density) distribution obtained from equations (8) and (9), bearing in mind equation (2). The total or aggregate ASD of the force due to a crowd of imperfect pedestrians can then be found using a joint distribution function (Newland 1993).

Firstly, two uncorrelated random walking force signals $p_{1}(t)$ and $p_{2}(t)$ from two pedestrians are considered. The mean square of the sum of the two signals is given by:

$$
\begin{aligned}
& E\left[\left(p_{1}(t)+p_{2}(t)\right)^{2}\right]=E\left[p_{1}^{2}(t)+p_{2}^{2}(t)+2 p_{1}(t) p_{2}(t)\right] . \\
& =E\left[p_{1}^{2}(t)\right]+E\left[p_{2}^{2}(t)\right]+2 E\left[p_{1}(t) p_{2}(t)\right]
\end{aligned}
$$

and, since $p_{1}(t)$ and $p_{2}(t)$ are assumed to be uncorrelated, $E\left[p_{1}(t) p_{2}(t)\right]=0$. Hence, the mean square of the sum of the two signals is the sum of the mean square of each signal. Also, by extension, the total mean square of $N$ uncorrelated signals of equal mean square is $N$ times the mean square of a single signal and, naturally, the $R M S$ will then be 
proportional to $\sqrt{N}$. Consequently, if we consider $p_{i}(t)$ to be narrow band random signals, it follows that the total ASD of uncorrelated signals $(i=1,2, \ldots, N)$ also goes with $\sqrt{N}$.

For an individual $i$ with weight $W$ and mean pacing rate $\bar{f}_{i}$, the ASD of footfall forces for the $n$th harmonic can be obtained as:

$$
S_{F, n}(f)=\left[W G_{n}\left(\bar{f}_{i}\right)\right]^{2} S_{G_{n}^{\prime}}\left(f / \bar{f}_{i}\right)
$$

For a crowd of $N$ pedestrians, the broadband contribution $d S_{P, n}(f)$ to mean square of loading in the $n$th harmonic due to the proportion $N \phi(\bar{f}) d \bar{f}$ of pedestrians with pacing rates in the narrow range $\bar{f} \pm d \bar{f} / 2$ will then be:

$$
d S_{P, n}(\bar{f})=N \phi(\bar{f}) S_{F, n}(\bar{f}) d \bar{f}=N\left[W G_{n}(\bar{f})\right]^{2} \cdot S_{G_{n}^{\prime}}(f / \bar{f}) \cdot(\phi(\bar{f}) / n) \cdot d \bar{f}
$$

It should be noticed that in equation (13) the division by $n$ compensates for the broadening of $\phi(\bar{f})$ when applied to $n>1$, since $\phi(\bar{f})$ scales to a larger number of spectral lines of ASD. The total ASD, $S_{P, n}(f)$, of loading at the $n$th harmonic due to a 
crowd of $N$ imperfect and unsynchronised pedestrians with mean weight $W$ and whose mean pacing rates range from $\bar{f}_{1}$ to $\bar{f}_{2}$ will then be given by:

$$
S_{P, n}(f)=(N / n) \int_{\bar{f}_{1}}^{\bar{f}_{2}}\left[W G_{n}(\bar{f})\right]^{2} \cdot S_{G_{n}^{\prime}}(f / \bar{f}) \cdot \phi(\bar{f}) d \bar{f}
$$

Because of the way it is normalised, the area under the ASD function $S_{G_{n}^{\prime}}(f / \bar{f})$ must be exactly 0.5 for each harmonic even if it collapses to a single spectral line. Therefore, equation (14) reduces to:

$$
S_{P, n}(f)=(N / n) W^{2} \phi(f) \cdot G_{n}^{2}(f) / 2
$$

if each pedestrian walks perfectly at his/her own pacing frequency.

Figure 12 shows the aggregate root ASD $S_{P, n}(f)$ for 6 harmonics of walking obtained via equation (15) by assuming the following statistics for walking paces for groups of pedestrians: $\mu_{\bar{f}}=2 \mathrm{~Hz}$ and coefficient of variation of the mean pacing rate $\operatorname{COV}(\bar{f})=0.1$. The values are in line with data provided by Matsumoto et al. (1978) and Murray et al. (1964). The difference obtained by applying equation (14) instead of equation (15) turns out to be small unless the COV is much reduced, hence it is proposed to use equation (15) for crowd loading. 
The ASD presented in Figure 12 is normalised by total weight of pedestrians. The values used are based on the test data described in this paper for the three test subjects for which it was established that: $G_{1}=0.37 \bar{f}-0.42, G_{2}=0.053, G_{3}=0.042$, $G_{4}=0.041, G_{5}=0.027$ and $G_{6}=0.018$. These values, and any frequency dependence, are in line with reported values (Young, 2001), except that $G_{2}$ is found to be significantly smaller, while $G_{1}$ is larger in this study.

\section{Coherency of loading and effect of mode shape}

The above analysis has not taken into account the vibration mode shape and correlation (i.e. synchronisation) between pedestrians. To account for mode shape, the frequency domain approach applied to analysis of vertical vibrations of the Millennium Bridge in London (Dallard et al. 2001) used a factor on ASD involving squared mode shape. A more thorough approach would adopt a procedure similar to that used to study dynamic effects of turbulent winds on linear structures (Simiu and Scanlan 1996) which will be outlined here.

For two random loads $p_{1}(t)$ and $p_{2}(t)$ applied along a linear structure at degrees of freedom (DOFs) $z_{1}$ and $z_{2}$ having modal shape ordinates $\psi_{z_{1}}$ and $\psi_{z_{2}}$ and having ASDs $S_{P_{1}}(f)$ and $S_{P_{2}}(f)$, the ASD of response at a third DOF $z$ is given by (Simiu and Scanlan 1996): 


$$
\begin{aligned}
& S_{X}(f)=\left|\psi_{z_{1}} \psi_{z} H(f)\right|^{2} S_{P_{1}}(f)+\left|\psi_{z_{2}} \psi_{z} H(f)\right|^{2} S_{P_{2}}(f) \\
& +2\left(\psi_{z} \psi_{z_{1}}\right)\left(\psi_{z} \psi_{z_{2}}\right)|H(f)|^{2} S_{P_{1} P_{2}}(f)
\end{aligned}
$$

where, in mode $r$ with modal mass $m_{r}$, natural frequency $f_{r}$ and modal damping ratio $\zeta_{r}$, the frequency response function for acceleration response is the product of inverse of modal mass and dimensionless dynamic amplification factor :

$$
H(f)=\frac{1}{m_{r}} \times \frac{-f^{2}}{f_{r}^{2}\left(1-f^{2} / f_{r}^{2}+2 i \zeta_{r} f / f_{r}\right)}
$$

The cross-spectral density $S_{P_{1} P_{2}}(f)$ is related to ASD of the separate signals via the coherence function $\operatorname{coh}(f)$ (McConnell 1995) which expresses correlation $\operatorname{coh}(f)$ between two signals as a function of frequency which satisfies:

$$
\left|S_{P_{1} P_{2}}(f)\right|^{2}=\operatorname{coh}(f) \cdot S_{P_{1}}(f) S_{P_{2}}(f)
$$

For two perfectly correlated loads $\operatorname{coh}(f)=1$, and assuming the signals have the same statistical properties so that $S_{P_{1}}(f)=S_{P_{2}}(f)$, the ASD of the corresponding response is:

$$
S_{X}(f)=|H(f)|^{2}\left(\psi_{z}\right)^{2} S_{P_{1}}(f)\left\{\psi_{z_{1}}^{2}+\psi_{z_{2}}^{2}+2 \psi_{z_{1}} \psi_{z_{2}}\right\}
$$


For completely uncorrelated loads where $S_{P_{1}}(f)=S_{P_{2}}(f)$ still applies, $\operatorname{coh}(f)=0$ so:

$$
S_{X}(f)=|H(f)|^{2}\left(\psi_{z}\right)^{2} S_{P_{1}}(f)\left\{\psi_{z_{1}}^{2}+\psi_{z_{2}}^{2}\right\}
$$

In the former case, if $\psi_{z_{1}}=\psi_{z_{2}}$ the ASD of response $S_{X}(f)$ is four times its counterpart for a single load, but where $\psi_{z_{1}}=-\psi_{z_{2}}$ the effects cancel. In the latter case of zero correlation the ASD is doubled if $\psi_{z_{1}}=\psi_{z_{2}}$, but also if $\psi_{z_{1}}=-\psi_{z_{2}}$.

A more realistic representation of groups of pedestrians is as distributed loads with limited correlation (i.e. $0<\operatorname{coh}\left(f, z_{1}, z_{2}\right)<1$ ). For example it has been observed that small groups of pedestrians often start walking approximately in step. The correlation and distribution of such loading is analogous to turbulent buffeting wind loads on a linear structure where cross-spectral density for two loads applied at two spatially separated points is assumed to diminish exponentially with increasing separation via a coherence function $\operatorname{coh}\left(f, z_{1}, z_{2}\right)$.

If a total weight $N W$ of $N$ perfect pedestrians is distributed over length $L$ then the ASD per unit length for the fundamental harmonic of walking loads is:

$$
S_{P, 1}(f)=(N W / L)^{2} \phi(f) \cdot G_{1}^{2}(f) / 2
$$


and the total response will be:

$$
S_{X}(f)=\psi_{z}^{2}|H(f)|^{2} S_{P, 1}(f) \int_{0}^{L} \int_{0}^{L} \psi_{z_{1}} \psi_{z_{2}} \operatorname{coh}\left(f, z_{1}, z_{2}\right) d z_{1} d z_{2}
$$

For computational reasons and also because each pedestrian can be modelled as a discrete vertical point load, the integral of equation (22) would be better evaluated as a discrete sum, with $\Delta z_{1}=\Delta z_{2}=L / N$ as the average length occupied by a single pedestrian in which case, for zero coherence and a sinusoidal mode shape, the value of the integral is $L^{2} / 2 N$

The discussion of equations (16)-(20) shows that for perfectly correlated pedestrians occupying a linear structure and exciting a symmetric mode, ASD of response is proportional to $N^{2}$ whereas for uncorrelated walking it is proportional to $N$. Depending of the value of $\operatorname{coh}\left(f, z_{1}, z_{2}\right)$ the true result will lie somewhere between the two extremes.

Although some studies on coherency, which concerns relative phase angles between pedestrians, were done in the past (Ebrahimpour and Fitts 1996), the exact nature of the walking coherence function $\operatorname{coh}\left(f, z_{1}, z_{2}\right)$ with respect to frequency and separation is still unknown and requires further research. 


\section{Validation of proposed analysis framework}

By its very nature, it is a challenge to provide a clear and comprehensive experimental proof for the analytical procedure proposed. However, a limited verification of the crowd loading model is based on data from a controlled crowd loading test on a footbridge in Singapore. The bridge has a fundamental mode at $1.64 \mathrm{~Hz}$ with a modal mass of approximately $150 \times 10^{3} \mathrm{~kg}$ corresponding to the unity scaled mode shape and modal damping ratio of approximately $0.7 \%$ when occupied by a crowd of moving pedestrians. The dynamic testing of the bridge was performed in a manner similar to the testing of the Millennium Bridge in London (Dallard et al. 2001) where pedestrians numbers were gradually increased while measuring continuously acceleration response.

Figure 13 shows the peak RMS acceleration response levels, having an observable dependence on pedestrian numbers. Equation (22) can be used to estimate the RMS response for $N=150$ pedestrians as follows, and as illustrated in Figure 14. Assuming zero coherence (i.e. no synchronisation between pedestrians), a normal distribution of pacing rates $\phi(\bar{f})$ with $\mu_{\bar{f}}=2 \mathrm{~Hz}$ and $\sigma_{\bar{f}}=0.2 \mathrm{~Hz}$, as described in equation (10), and assuming mean pedestrian weight of $735 \mathrm{~N}$, while accounting for mode shape, leads to estimated $R M S$ acceleration of $0.024 \mathrm{~m} / \mathrm{sec}^{2}$. This figure is a little lower than the maximum response $\left(0.033 \mathrm{~m} / \mathrm{sec}^{2} \mathrm{RMS}\right)$ achieved with maximum number of pedestrians, $N=150$, indicating a very low level of synchronisation between pedestrians, matching visual observations made during the test. 
For comparison, the maximum simulated response for the BD37/01 (BSI 2001) 'perfect pedestrian' generating a perfect $180 \mathrm{~N}$ amplitude sinusoidal force at $1.64 \mathrm{~Hz}$ and walking at $1.48 \mathrm{~m} / \mathrm{sec}$ would generate peak response of $0.07 \mathrm{~m} / \mathrm{sec}^{2}$, equivalent to $0.05 \mathrm{~m} / \mathrm{sec}^{2}$ $R M S$, even larger than observed for 150 real pedestrians.

The zero correlation for vertical walking forces is consistent with observations from Millennium Bridge in London (Dallard et al. 2001). It is notable that up to a certain and quite large number of pedestrians, the response due to their normal walking (i.e. random loading) is no more than for the single perfect pedestrian, an observation made many years ago by Wheeler (1982) and others.

\section{Conclusions}

A frequency domain approach has been developed for modelling response of long flexible structures, such as footbridges and long-span floors, to loads induced by one or more pedestrians. Explicit modelling of the effect of groups of pedestrians currently does not feature in the UK or Canadian footbridge design codes of practice, which are probably amongst the most advanced in the world. Therefore, the approach presented in this paper can serve as a framework for a more thorough and realistic treatment of vertical forces induced by groups of normally walking pedestrians which could be adopted in the design practice.

The first refinement of design procedures would account for the imperfection of individual walking excitation. This excitation is conventionally assumed in the design 
guidelines to be perfectly periodic and represented as a truncated Fourier series. While the fundamental harmonic of such loading is apparently hardly affected, for higher harmonics coinciding with frequencies of vibration modes, the 'leaking' of energy into adjacent frequencies generally results in reduced response compared with the prediction using a 'perfect pedestrian' model.

The second refinement follows principles used in the spectral approach to wind loading, accommodating any correlation between pedestrians as well as the statistical distribution of pacing rates in a walking crowd. The resulting response predictions are necessarily given as RMS rather than peak values, and this is consistent with commonly used vibration acceptance criteria.

One case study implies that for normal walking, the degree of correlation of vertical walking forces between pedestrians approaches zero and that existing code provisions using a single perfect pedestrian can account for the effect of large, but not excessively large, crowds. In the case where a more realistic assessment of the effect of low level pedestrian traffic is needed, the proposed walking excitation model could be used.

Finally, if this framework, which has been demonstrated to be feasible and reasonable, is to be adopted and further developed, more research work is required to verify it against results from experiments on real full-scale structures, and to determine the likely values 
of coherence function $\operatorname{coh}\left(f, z_{1}, z_{2}\right)$ as a measure of synchronisation between pedestrians. 


\section{References}

Bachmann, H., and Ammann, W. 1987. Vibrations in structures induced by man and machine. Structural Engineering Documents 3e, IABSE, Switzerland.

Bachmann, H., Pretlove, A.J., and Rainer, H. 1995. Vibration problems in structures practical guidelines: Appendix G - Dynamic forces from rhythmical human body motions. Birkhäuser, Basel, Switzerland.

Barnett, S., Cunningham, J.L., and West, S. 2001. A comparison of vertical force and temporal parameters produced by an in-shoe pressure measuring system and a force platform. Clinical Biomechanics, 16: 353-357.

Belli, A., Bui , P., Berger, A., Geyssant, A., and Lacour, J-R. 2001. A treadmill ergometer for three-dimensional ground reaction forces measurement during walking. Journal of Biomechanics, 34: 105-112.

BSI 1992. BS6472 - Evaluation of human exposure to vibration in buildings (1 Hz to $80 \mathrm{~Hz}$ ). British Standards Institution. Milton Keynes, UK.

BSI 2001. Steel, concrete and composite bridges. Specification for loads, BS5400: Part 2 (BD37/01, Appendix B). British Standards Institution. Milton Keynes, UK. 
CSA 2000. Canadian Highway Bridge Design Code CAN/CSA-S6-00. Canadian Standards Association

Dallard, P., Fitzpatrick, A.J., Flint, A., Le Bourva, S., Low, A., Ridsdill Smith, R.M., and Willford, M. 2001. The London Millennium footbridge. The Structural Engineer, 79: 1733.

Ebrahimpour, A., and Fitts, L.L. 1996. Measuring coherency of human-induced rhythmic loads using force plates. ASCE Journal of Structural Engineering, 122: 829-831.

Ebrahimpour, A., Hamam, A., Sack, R.L. and Patten, W.N. 1996. Measuring and modelling dynamic loads imposed by moving crowds. ASCE Journal of Structural Engineering, 122: 1468-1474.

Ellis, B. 2000. On the response of long-span floors to walking loads generated by individuals and crowds. The Structural Engineer, 78: 17-25.

Eriksson, P-E. 1994. Vibration of low-frequency floors -dynamic forces and response prediction. Doctoral thesis, Publication D 94:3, Chalmers University of Technology, Goteborg, Sweden. 
Forner Cordero, A., Koopman, H.J.F.M., Vander Helm, F.C.T. 2002. Estimation of the ground reaction forces and torques from the motion data and pressure insoles.

Proceedings of the Symposium on the 3-D analysis of human movement, Newcastle, UK, pp. $97-100$.

Gordon, C.G. 1991. Generic criteria for vibration-sensitive equipment. Proceedings of International Society for Optical Engineering, San Jose, CA, Vol. 1619, pp. 71-85.

Kerr, S. C. 1998. Human induced loading on staircases. PhD Thesis, University of London.

Matsumoto, Y., Nishioka, T., Shiojiri, H., and Matsuzaki, K. 1978. Dynamic design of footbridges. IABSE Proceedings P17/78, 1-15.

McConnell, K. 1995. Vibration Testing - Theory and Practice. John Wiley \& Sons, Inc., New York.

Mouring, S.E., and Ellingwood, B.R. 1994. Guidelines to minimize floor vibrations from building occupants. ASCE Journal of Structural Engineering, 120: 507-526.

Murray, M.P., Drought A.B., Kory R.C. 1964. Walking patterns of normal men. The Journal of Bone and Joint Surgery, 46A: 335-360. 
Newland, D.E. 1993. An introduction to random vibrations, spectral and wavelet analysis. Longman, Harlow, UK.

Pavic. A., and Reynolds, P. 2002. Vibration serviceability of long-span concrete building floors. Part 1: Review of background information'. The Shock and Vibration Digest, 34: 2002, 187-207.

Pimentel, R. L., Pavic, A., and Waldron, P. 2001. Evaluation of design requirements for footbridges excited by vertical dynamic forces from walking, Canadian Journal of Civil Engineering, 28: (5). 769-777.

Rainer, J. H., Pernica, G. and Allen, D. E. 1988. Dynamic loading and response of footbridges. Canadian Journal of Civil Engineering, 15: 66-71.

Rao, S., Yack, H.J., Hsu, M-J., Wahi, R., and Nielsen, D. 2002. Effect of prosthetic foot type and walking velocity on vertical ground reaction forces experienced by the sound and involved limbs in individuals with transtibial amputation. Proceedings of the Seventh Annual Meeting, Gait and Clinical Movement Analysis Society, Chattanooga, USA, April, www.utc.gait.edu/gait2002.

Razian M.A., Pepper M.G., 1998. A novel triaxial force transducer for in-shoe and other biomechanical applications. Journal of Biomechanics 31, Suppl 1, 133. 
Simiu, E., and Scanlan, R.H. 1996. Wind effects on structures, 3rd Edition. Wiley \& Sons, New York.

Smith, J.W. (1988). Vibration of structures - applications in civil engineering design. Chapman \& Hall, London.

Stevenage S.V., Nixon M.S., Vince K. 1999. Visual analysis of gait as a cue to identity. Applied Cognitive Psychology 13 (6): 513-526.

Wheeler, J.E. 1982. Prediction and control of pedestrian-induced vibration in footbridges. ASCE Journal of Structural Engineering, 108: 2045-2065.

Winter, D. A. (1990) Biomechanics and motor control of human movement, $2^{\text {nd }}$ Edition. Wiley \& Sons, New York.

Woodburn, J., and Helliwell, P.S. 1996. Observations on the f-scan in-shoe pressure measuring system. Clinical Biomechanics, 11: 301-304.

Young, P. 2001. Improved floor vibration prediction methodologies. Proceedings of Arup Vibration Seminar on Engineering for Structural Vibration - Current Developments in Research and Practice. IMechE, London. 


\section{Tables}

Table 1 Parameters fitting equation (8) to Fourier spectra of walking forces, for

$$
d \bar{f}=\bar{f} / 64
$$

\begin{tabular}{ccccc}
\hline Harmonic & $\mathrm{A}$ & $\mathrm{B}$ & $\mathrm{C}$ & $\mathrm{D}$ \\
\hline 1 & 0.014 & 0.845 & 0.647 & 0.0490 \\
\hline 2 & 0.119 & 0.615 & 1.213 & 0.0034 \\
\hline 3 & 0.083 & 0.418 & 1.126 & 0.0056 \\
\hline 4 & 0.064 & 0.431 & 1.172 & 0.0054 \\
\hline 5 & 0.083 & 0.427 & 1.309 & 0.0032 \\
\hline 6 & 0.115 & 0.364 & 1.674 & 0.0007 \\
\hline
\end{tabular}




\section{Figure captions}

Figure 1: $\quad$ Reconstruction of walking time series by replicating footfall force

Figure 2: Measurements using Gaitway instrumented treadmill. A fluorescent marker is visible on the right foot of the test subject.

Figure 3: $\quad$ Variation of footfall width and area (impulse, normalised with respect to weight) vs. average footfall (pacing) rate for three test subjects.

Figure 4: $\quad$ Comparison of real and synthetic force time series for 30 paces of walking at mean pacing rate $\bar{f}=1.91 \mathrm{~Hz}$, and response of $10^{3} \mathrm{~kg}$ oscillator with $1 \%$ damping and natural frequencies of $\bar{f}, 2 \bar{f}, 3 \bar{f}, 4 \bar{f}$. Envelop of responses corresponds to synthetic walking.

Figure 5: Response spectra corresponding to Figures 4,5 for peak and RMS responses resulting from synthetic (thin) and real walking loads (bold). Oscillator frequencies $\mathrm{f}[\mathrm{Hz}]$ range within $0.9 \bar{f}<f<1.1 \bar{f}$ where $\bar{f}=1.91 \mathrm{~Hz}$.

Figure 6: Maximum harmonic RMS response for simulated and real time series of 90 paces at a range of pacing rates $\bar{f}$ for $1 \%$ damping. Values at each $\bar{f}$ are obtained from plots such as Figure 4. Crest factors (CF) are given for input around each harmonic.

Figure 7: $\quad$ Maximum harmonic peak response for range of pacing rates $\bar{f}$ for real and simulated time series. Values at each $\bar{f}$ are obtained from plots such as Figure 4. 
Figure 8: $\quad$ Fourier amplitude (line) spectrum of real forces for $\bar{\omega}=1.911 \mathrm{~Hz}$. Frequency spacing is $\bar{f} / 64$.

Figure 9: Fourier amplitude spectrum simulated for $\bar{f}=1.91 \mathrm{~Hz}$. Frequency spacing is $\bar{f} / 64$.

Figure 10: Superimposed averaged harmonic Fourier amplitudes $G_{n}^{\prime}(f / \bar{f})$, normalised with respect to simulated values.

Figure 11: Averaged harmonic Fourier amplitudes $G_{n}^{\prime}(f / \bar{f})$, normalised with respect to simulated values and fitted to equation (8).

Figure 12: Aggregate square root ASD of multi-pedestrian loading for mean pace rate $\mu_{\bar{f}}=2 \mathrm{~Hz}$ and $\sigma_{\bar{f}}^{2}=0.2 \mathrm{~Hz}$ obtained from equation.

Figure 13: $\quad$ RMS response of footbridge in first torsional mode at $1.64 \mathrm{~Hz}$ with modal mass $150 \times 10^{3} \mathrm{~kg}$ due to up to 150 pedestrians.

Figure 14: Response of bridge mode at $1.64 \mathrm{~Hz}$ to first harmonic of walking with mean pacing rate $2 \mathrm{~Hz}$ computed using equations (15) and (22). RMS of total response is $0.024 \mathrm{~m} / \mathrm{sec}^{2}$. 


\section{Figures}

Figure 1: Reconstruction of walking time series by replicating footfall force

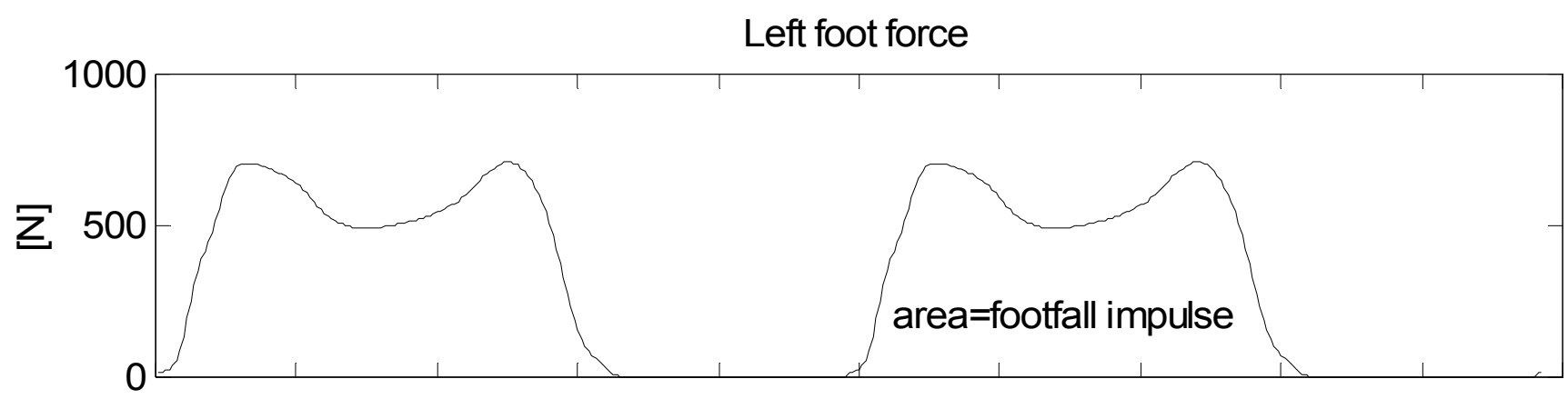

Right foot force
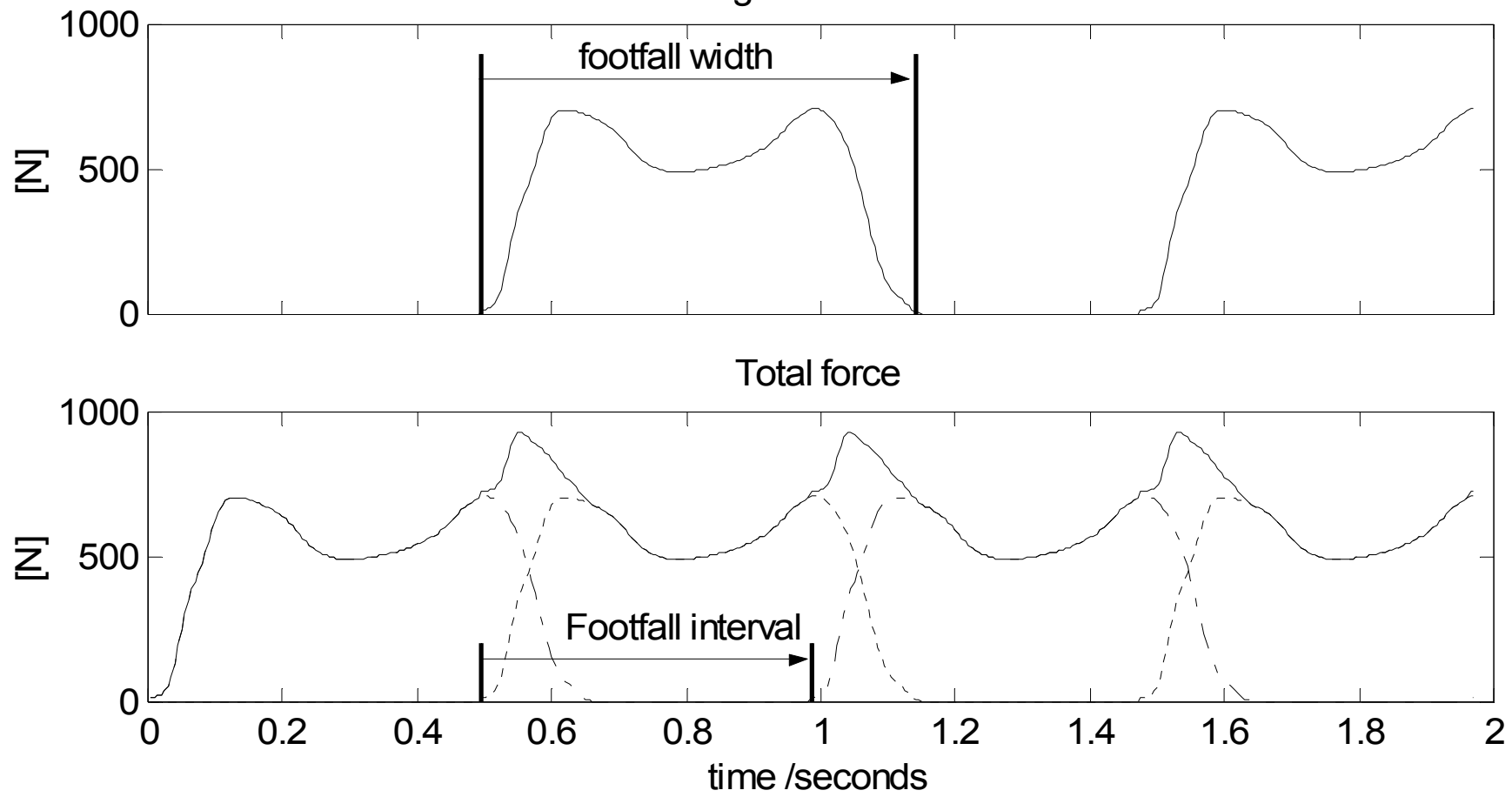
Figure 2: $\quad$ Measurements using Gaitway instrumented treadmill. A fluorescent marker is visible on the right foot of the test subject.

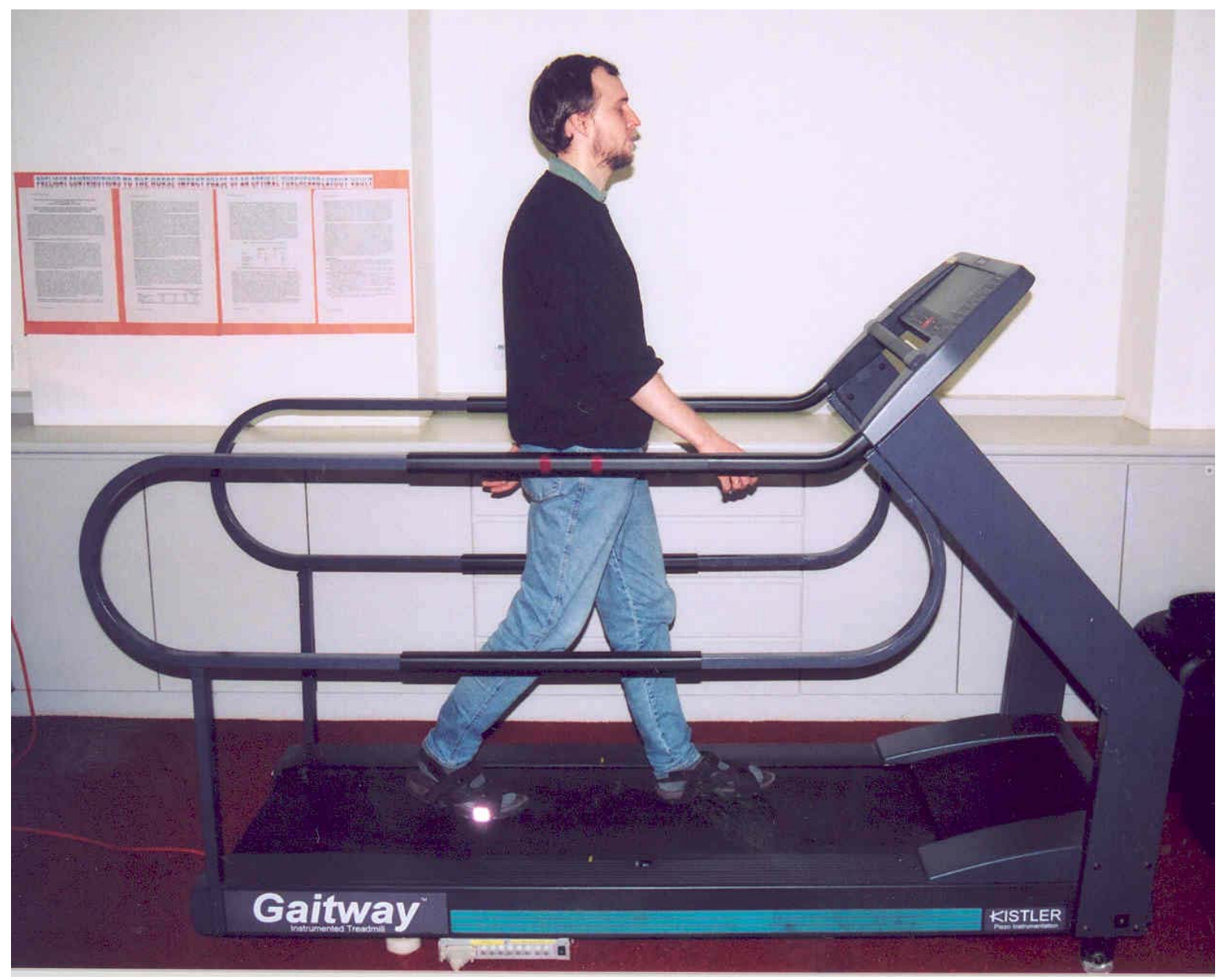


Figure 3: Variation of footfall width and area (impulse, normalised with respect to weight) vs. average footfall (pacing) rate for three test subjects.
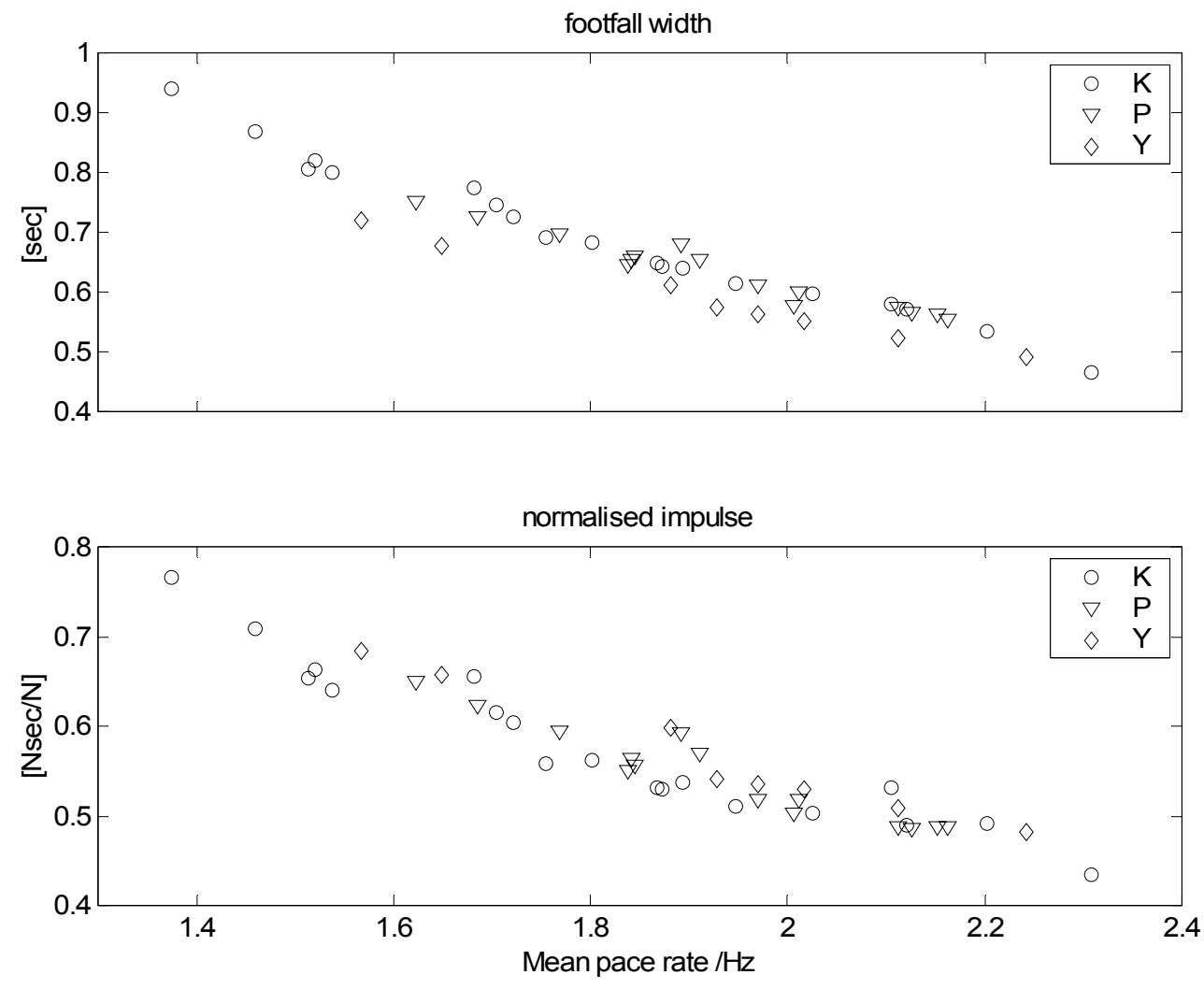
Figure 4: Comparison of real and synthetic force time series for 30 paces of walking at mean pacing rate $\bar{f}=1.91 \mathrm{~Hz}$, and response of $10^{3} \mathrm{~kg}$ oscillator with $1 \%$ damping and frequencies of $\bar{f}, 2 \bar{f}, 3 \bar{f}, 4 \bar{f}$. Envelop of responses corresponds to synthetic walking.
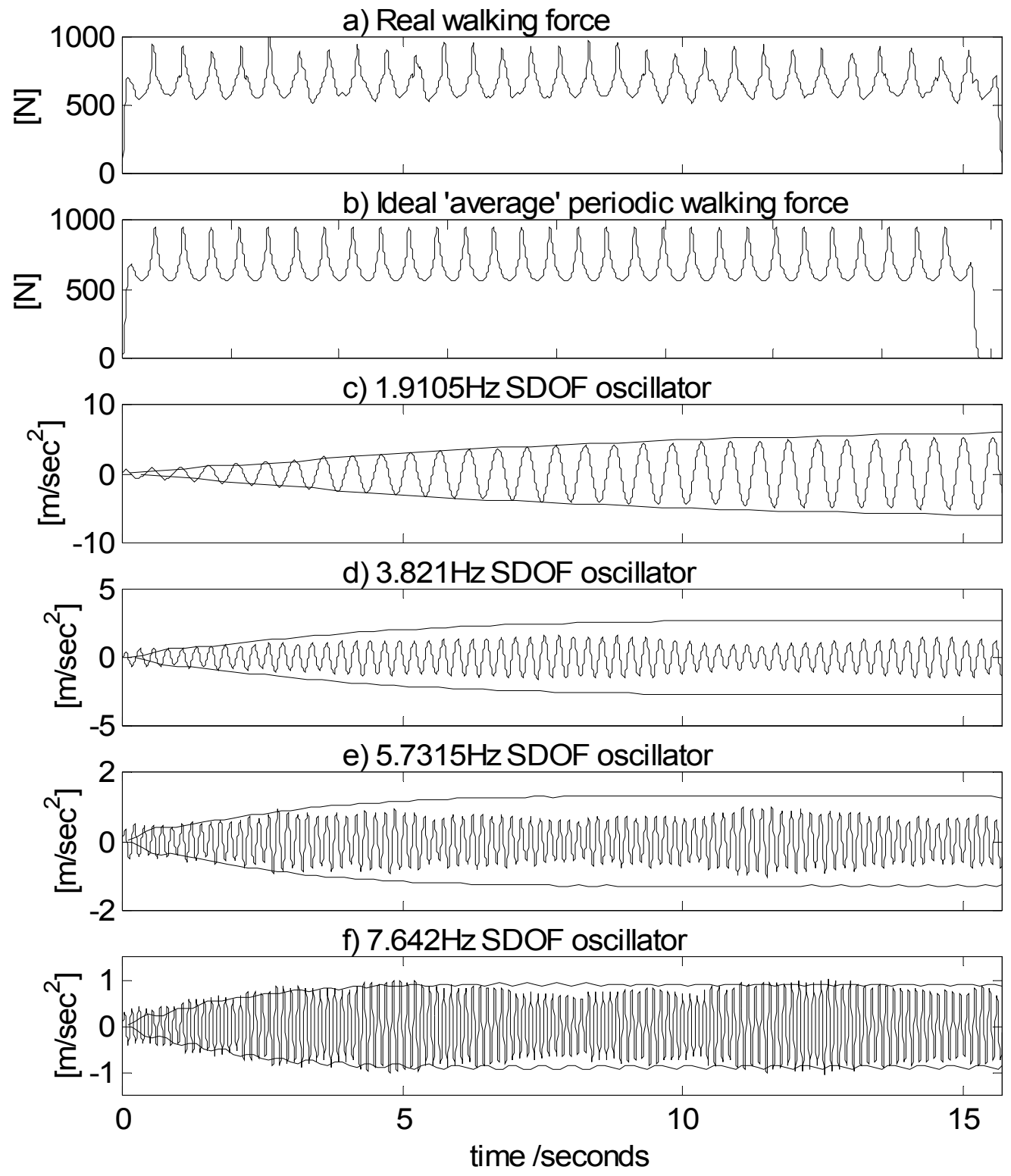
Figure 5: $\quad$ Response spectra corresponding to Figures 4,5 for peak and RMS responses resulting from synthetic (thin) and real walking loads (bold). Oscillator frequencies $\mathrm{f}[\mathrm{Hz}]$ range within $0.9 \bar{f}<f<1.1 \bar{f}$ where
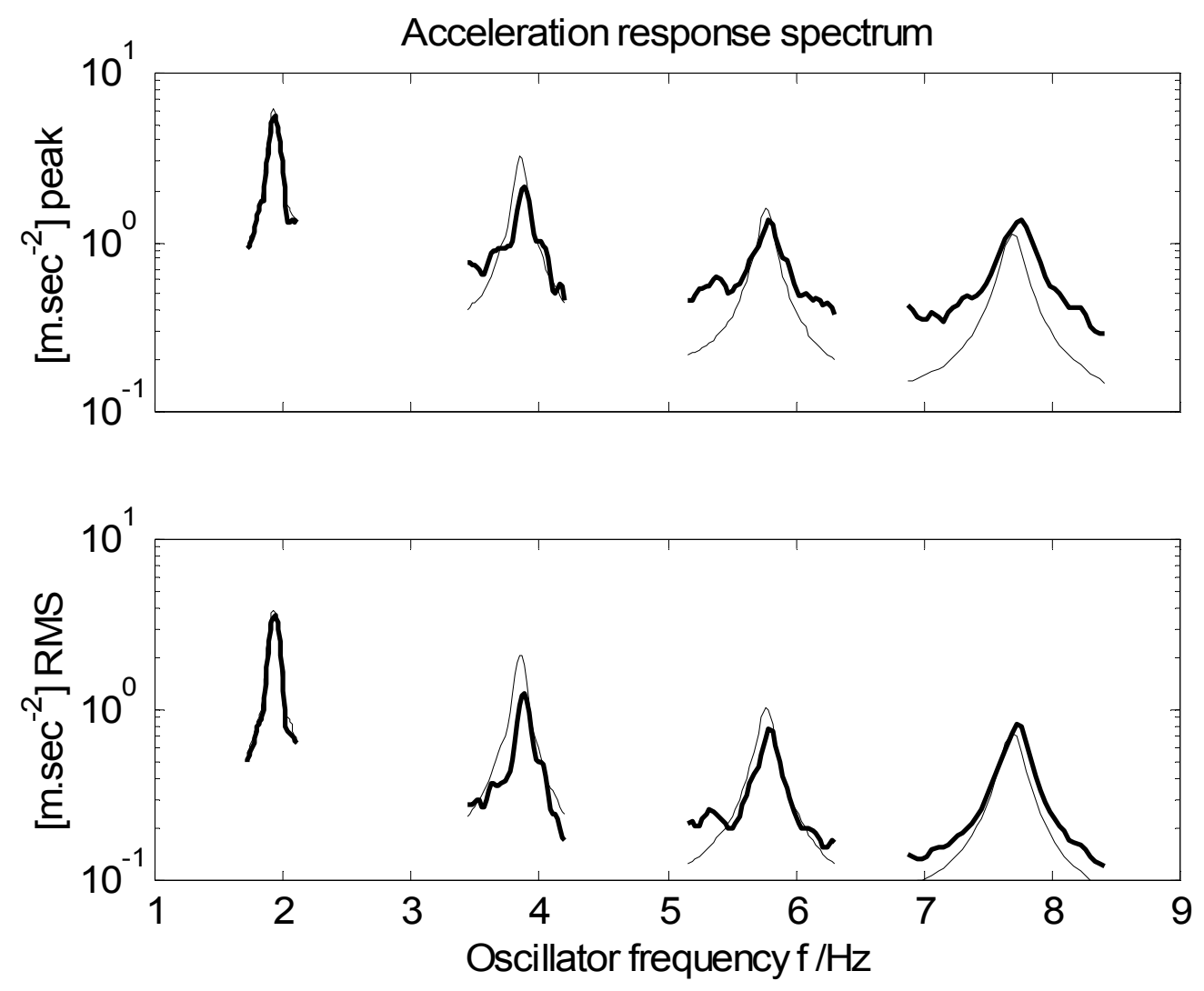

$\bar{f}=1.91 \mathrm{~Hz}$. 
Figure 6: Maximum harmonic RMS response for simulated and real time series of 90 paces at a range of pacing rates $\bar{f}$ for $1 \%$ damping. Values at each $\bar{f}$ are obtained from plots such as Figure 4. Crest factors (CF) are given for input around each harmonic.
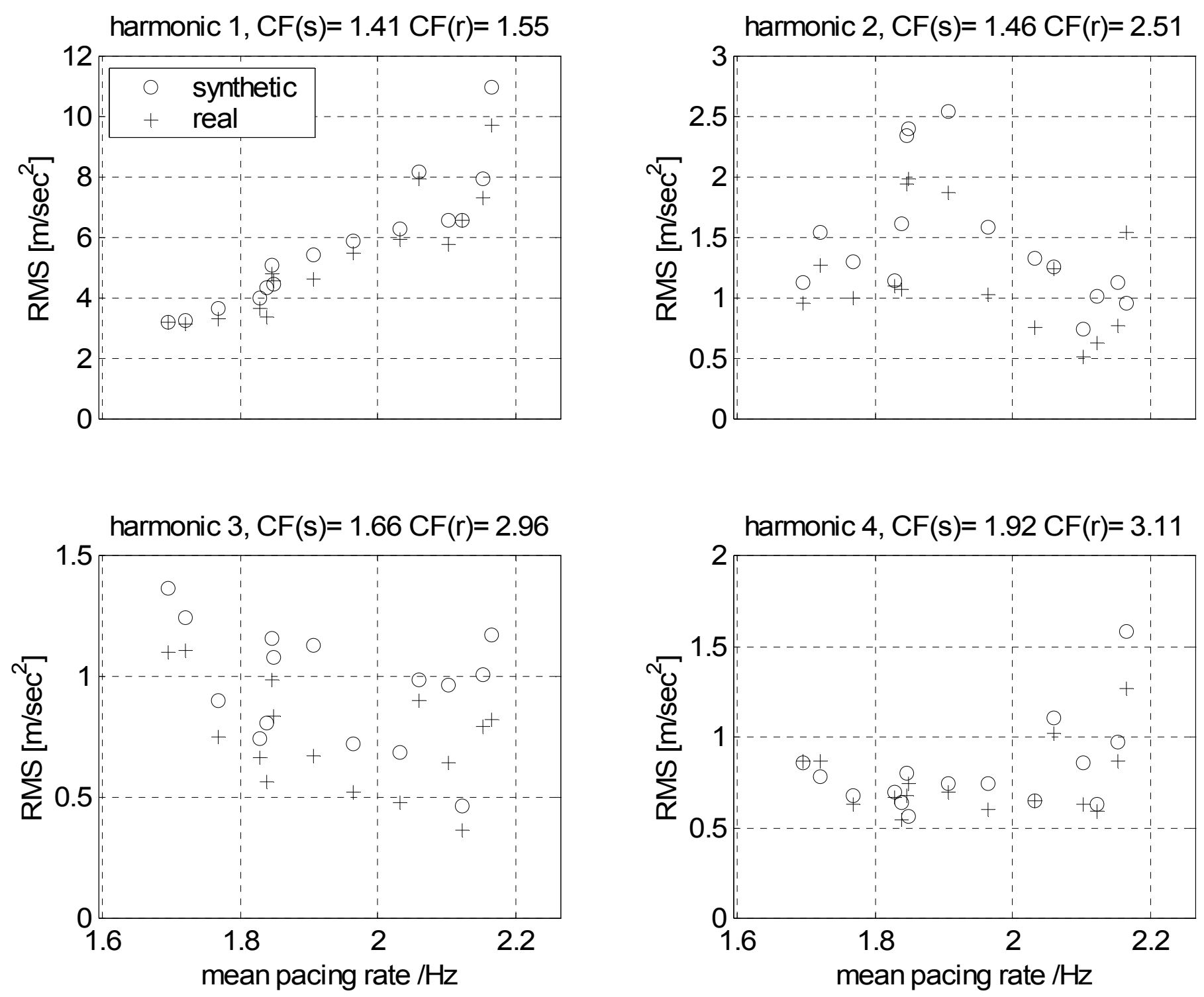
Figure 7: Maximum harmonic peak response for range of pacing rates $\bar{f}$ for real and simulated time series. Values at each $\bar{f}$ are obtained from plots such as Figure 4.
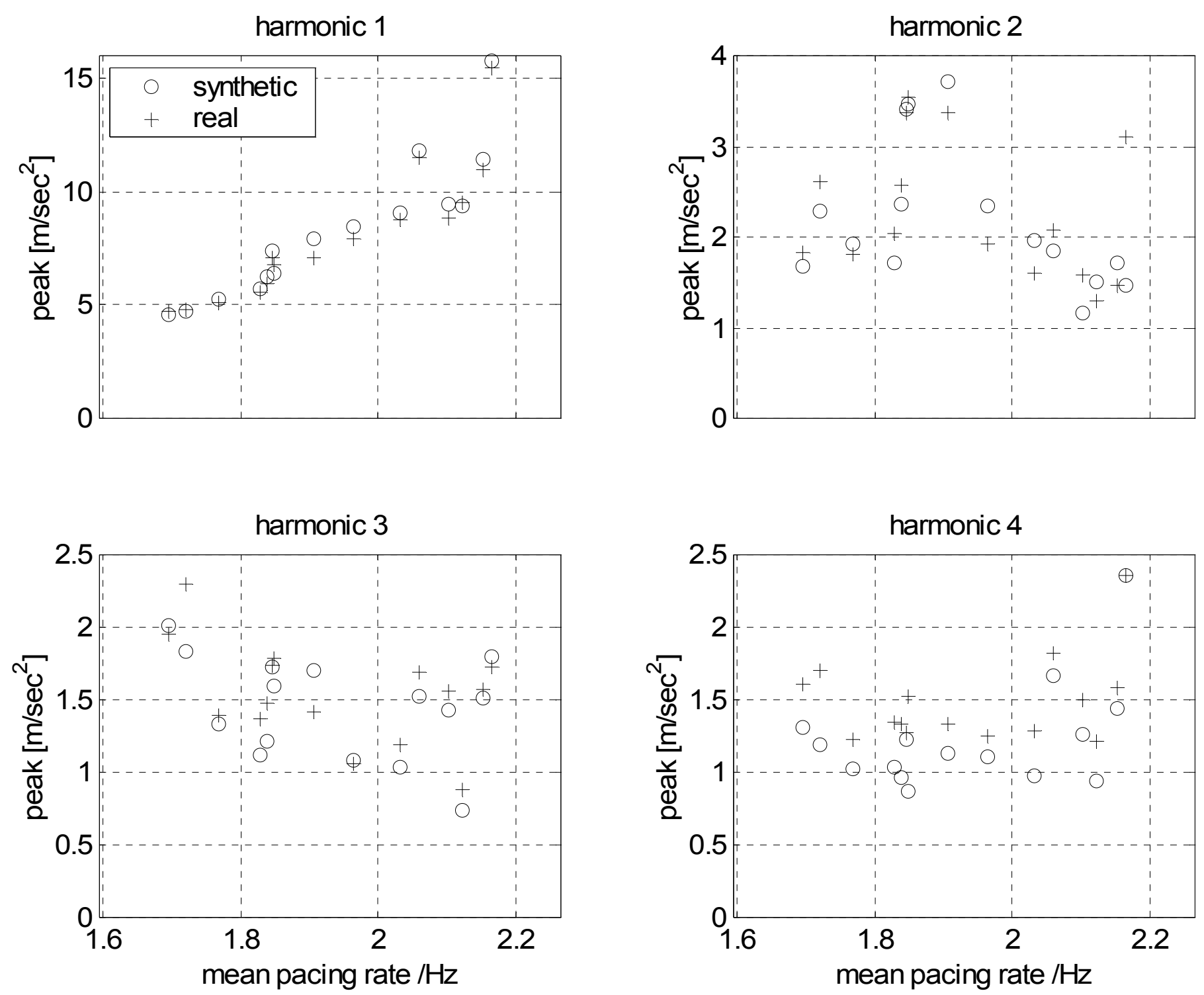
Figure 8: $\quad$ Fourier amplitude (line) spectrum of real forces for $\bar{f}=1.911 \mathrm{~Hz}$. Frequency spacing is $\bar{f} / 64$.

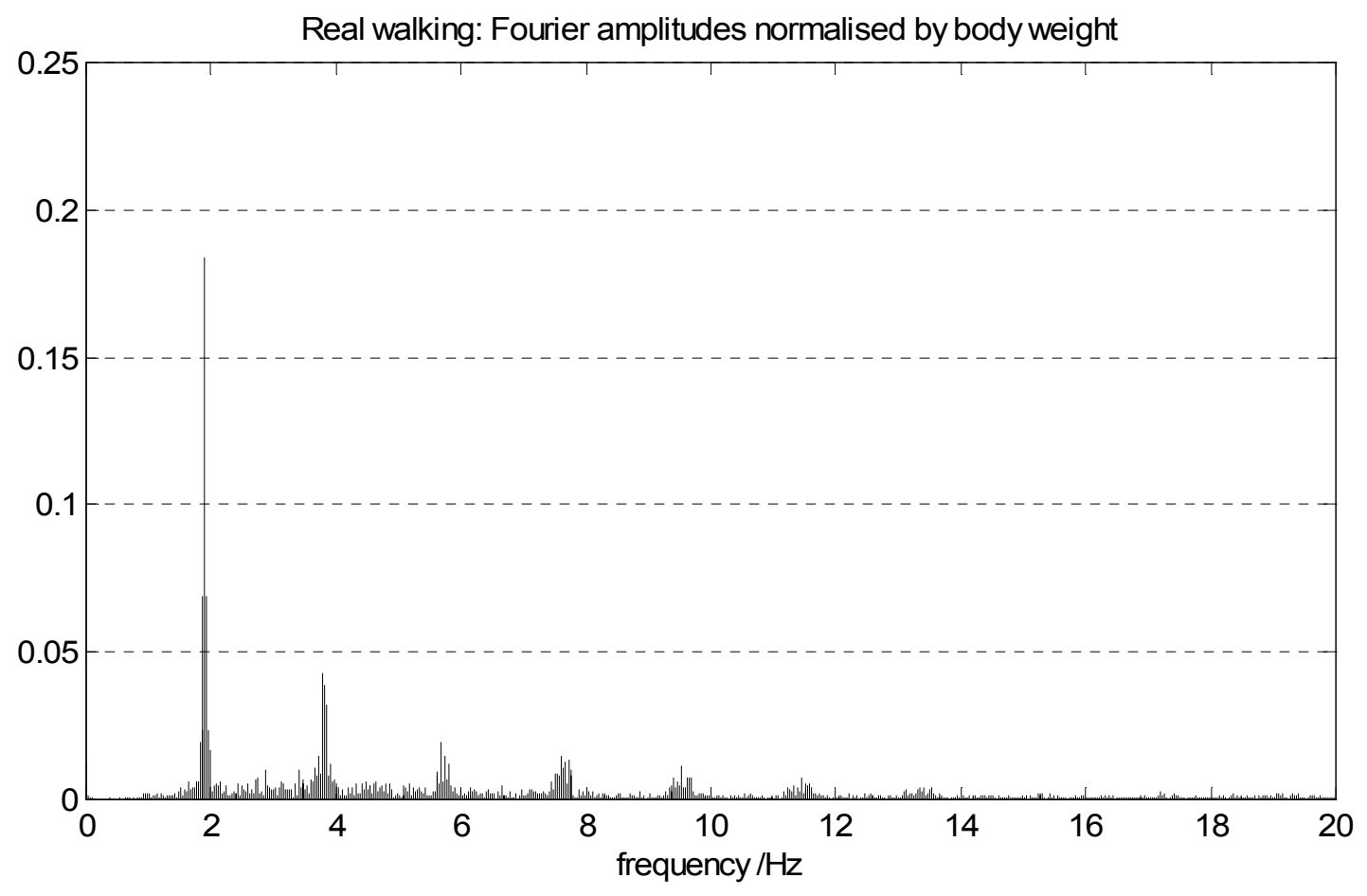


Figure 9: $\quad$ Fourier amplitude spectrum simulated for $\bar{f}=1.91 \mathrm{~Hz}$. Frequency spacing is $\bar{f} / 64$.

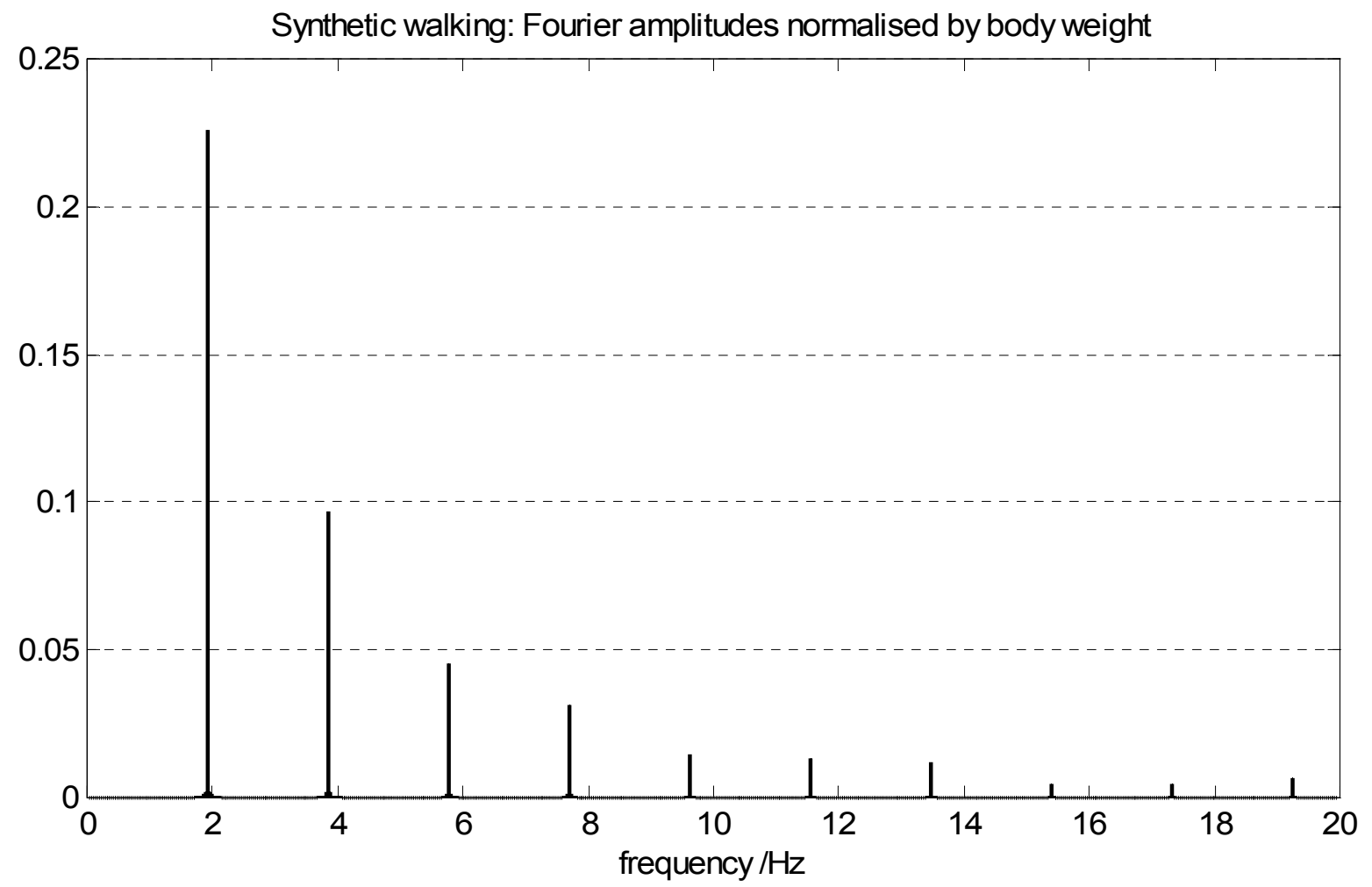


Figure 10: $\quad$ Superimposed averaged harmonic Fourier amplitudes $G_{n}^{\prime}(f / \bar{f})$, normalised with respect to simulated values.

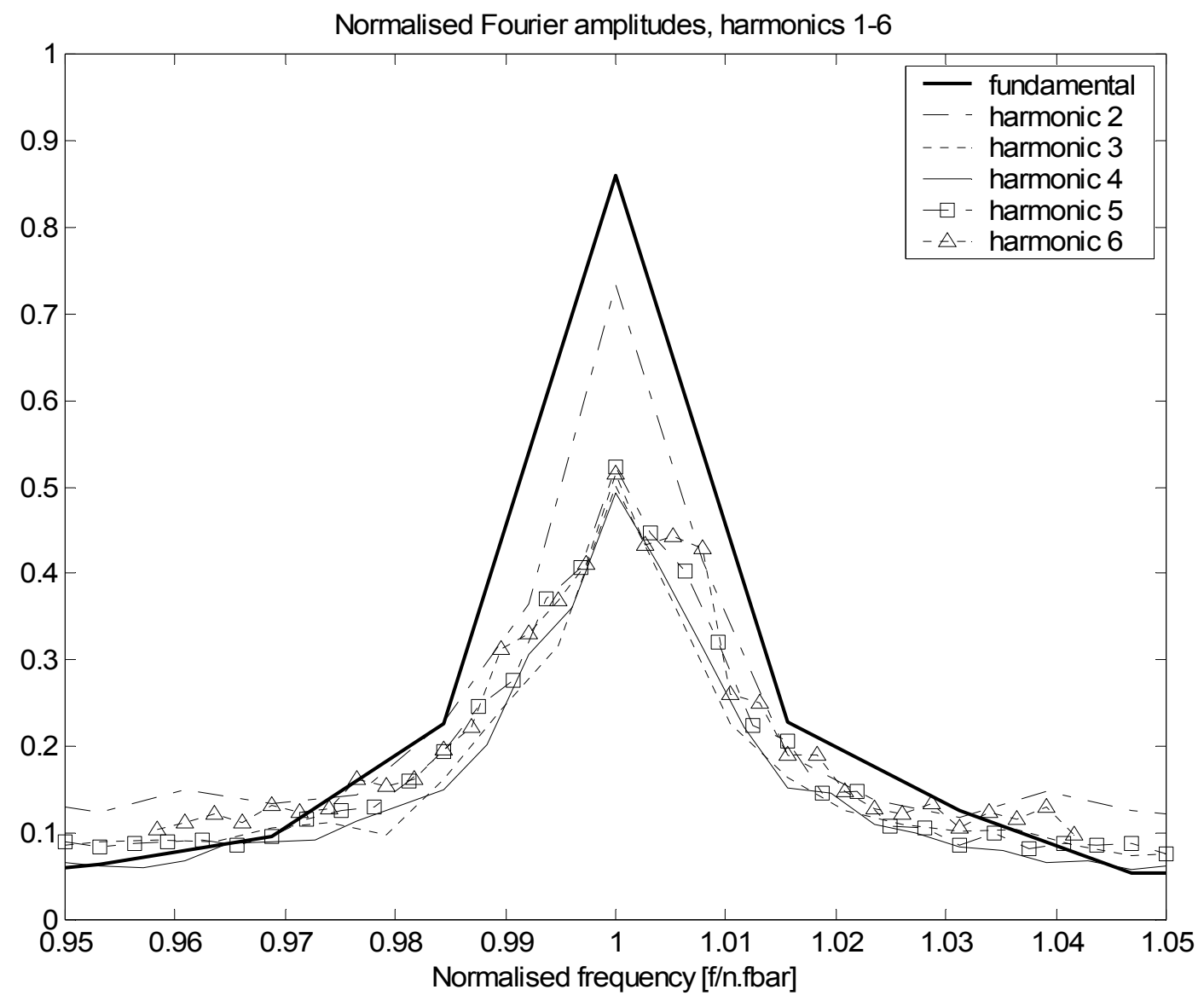


Figure 11: Averaged harmonic Fourier amplitudes $G_{n}^{\prime}(f / \bar{f})$, normalised with respect to simulated values and fitted to equation (8).

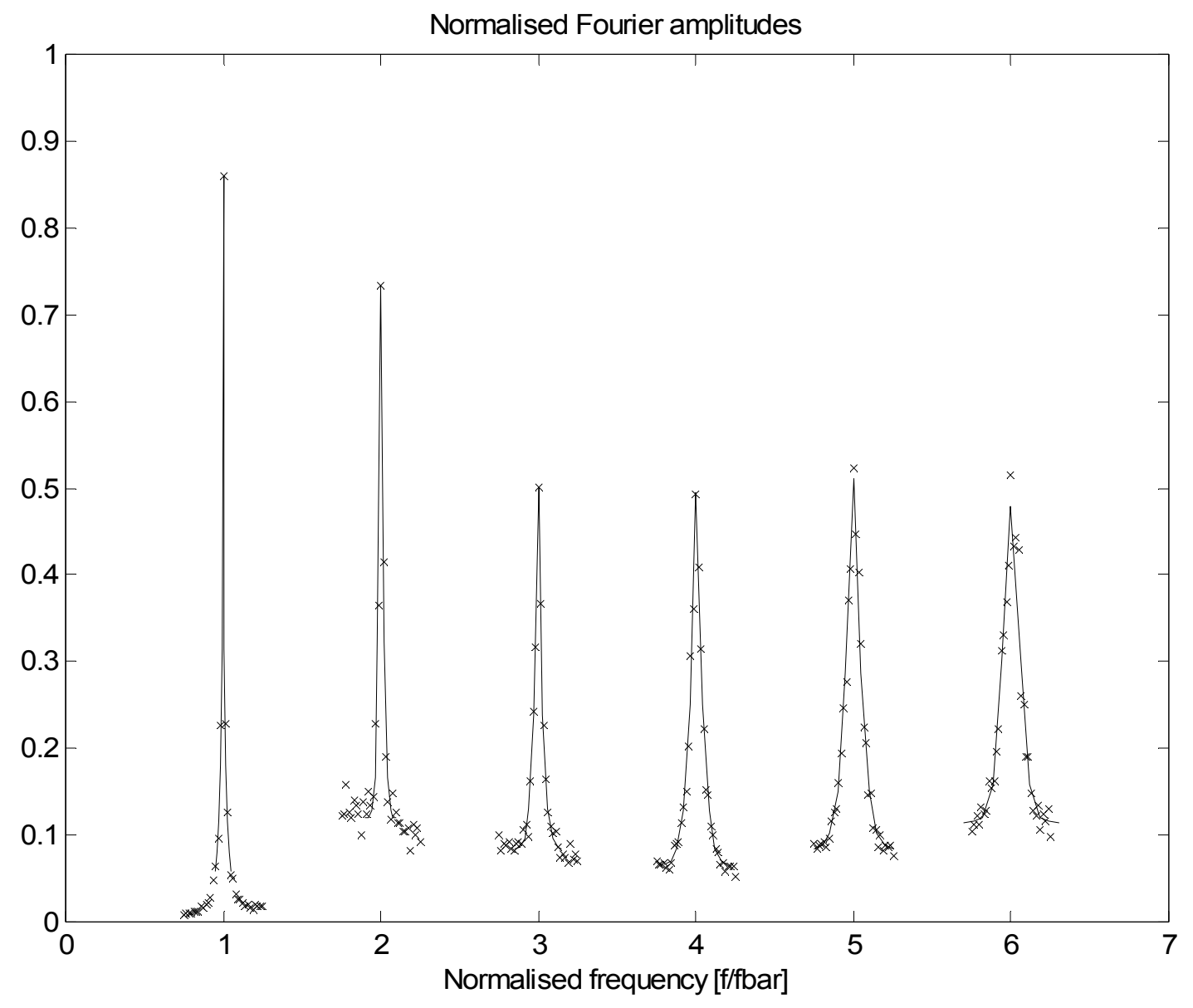


Figure 12: Aggregate square root ASD of multi-pedestrian loading for mean pace rate $\mu_{\bar{f}}=2 \mathrm{~Hz}$ and $\sigma_{\bar{f}}^{2}=0.2 \mathrm{~Hz}$ obtained from equation (15)

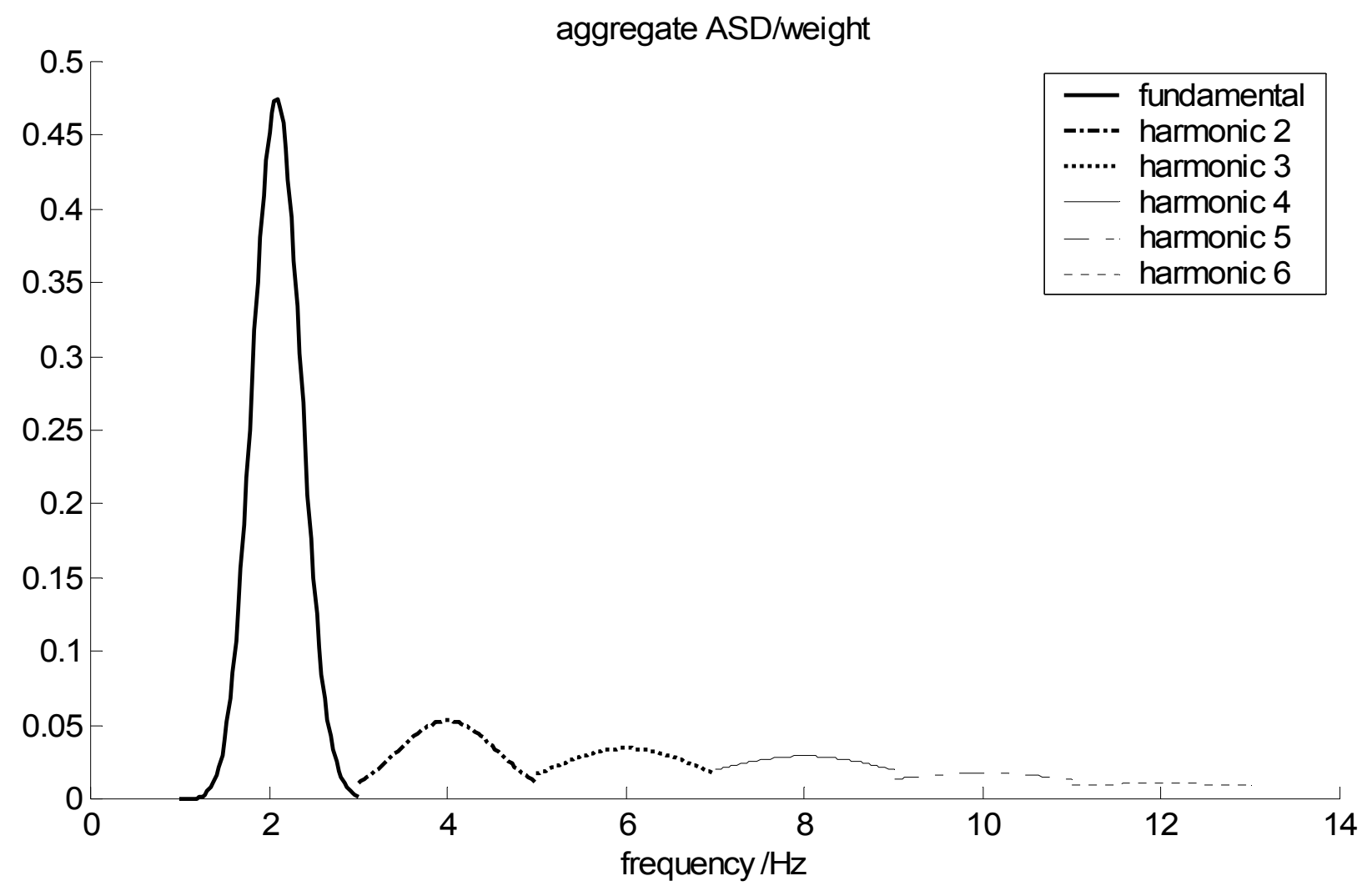


Figure 13: $\quad$ RMS response of footbridge in first torsional mode at $1.64 \mathrm{~Hz}$ with modal mass $150 \times 10^{3} \mathrm{~kg}$ due to up to 150 pedestrians.

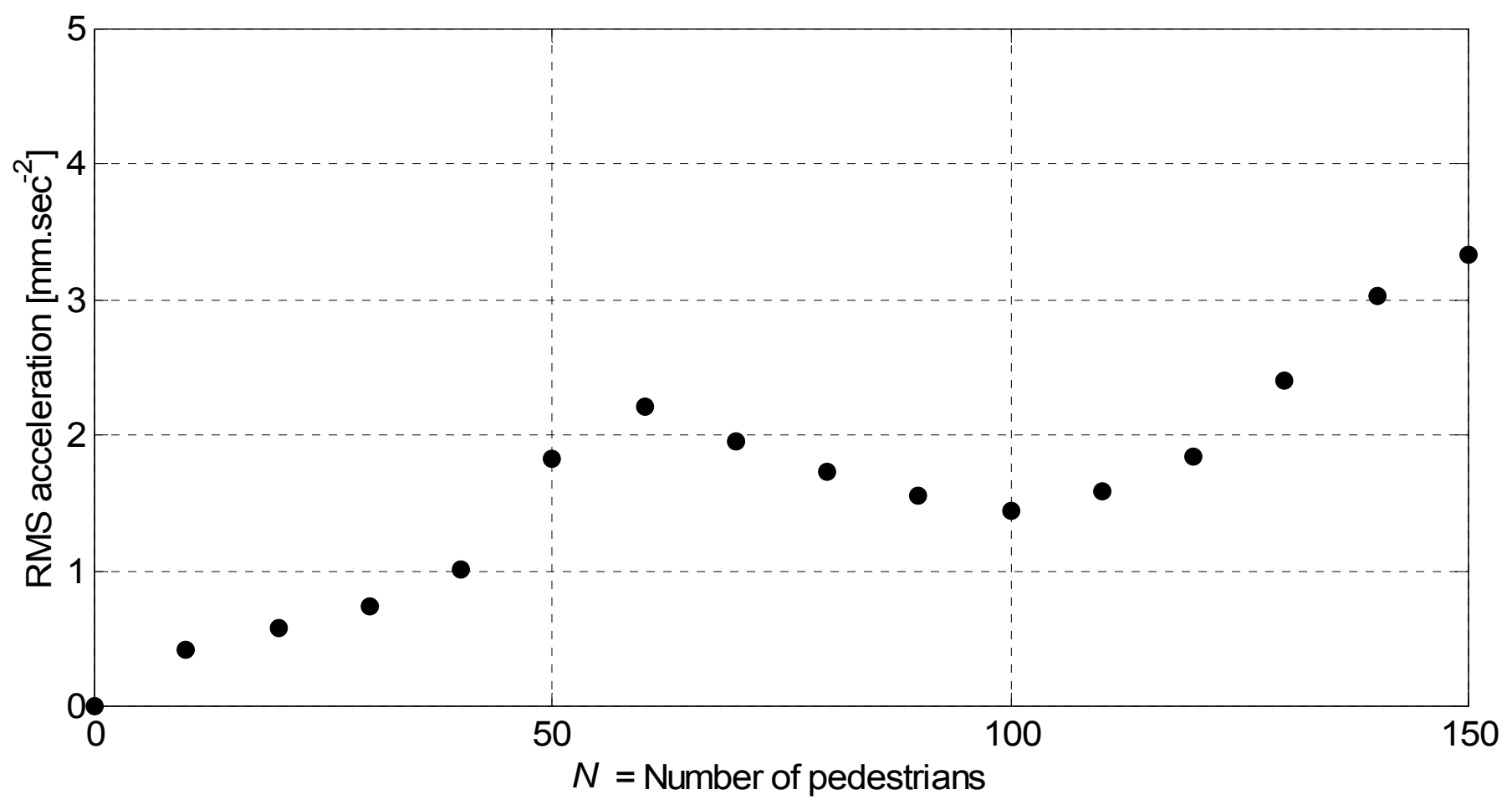


Figure 14: Response of bridge mode at $1.64 \mathrm{~Hz}$ to first harmonic of walking with mean pacing rate $2 \mathrm{~Hz}$ computed using equations (15) and (22). RMS of total response is $0.024 \mathrm{~m} / \mathrm{sec}^{2}$.
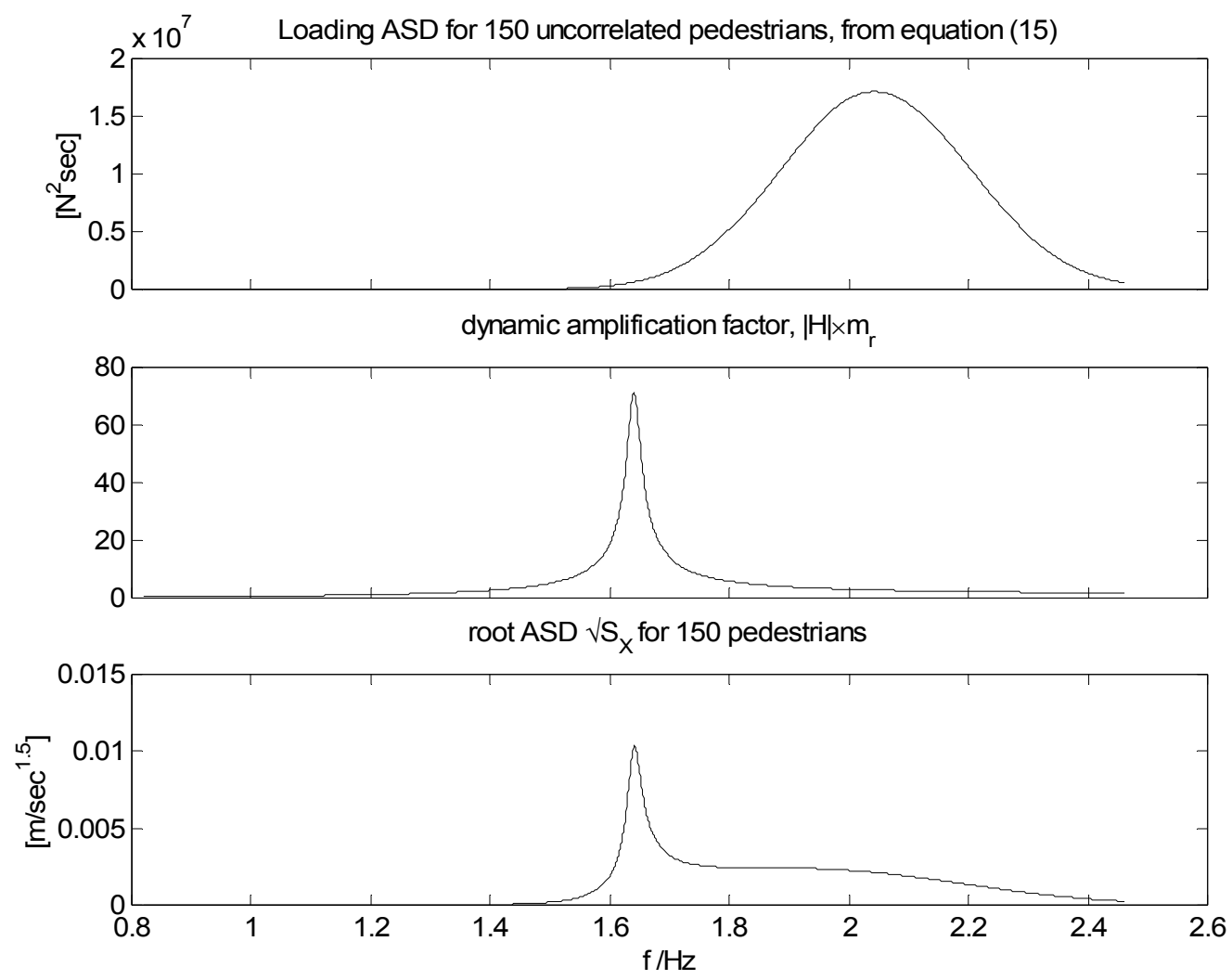